\title{
Differential transcriptomic response of Anopheles arabiensis to Plasmodium vivax and
} Plasmodium falciparum infection

Majoline Tchioffo Tsapi ${ }^{1,2, *}$, Etienne Kornobis ${ }^{3,4}$, Nicolas Puchot $^{1,2}$, Solomon English ${ }^{1,5}$, Caroline Proux $^{6}$, Jessy Goupeyou-Youmsi1 ${ }^{1,7 \# a}$, Anavaj Sakuntabhai ${ }^{1,2}$, Marie-Agnes-Dillies ${ }^{4}$ Randrianarivelojosia Milijaona ${ }^{8}$, Romain Girod ${ }^{9}$, Mamadou Ousmane Ndiath ${ }^{10 \# b}$, Catherine Bourgouin $^{1,2 *}$

${ }^{1}$ Functional Genetics of Infectious Diseases Unit, Institut Pasteur Paris, France

${ }^{2}$ CNRS UMR 2000 : Génomique évolutive, modélisation et santé (GEMS), Paris, France.

${ }^{3}$ Plate-forme Technologique Biomics - Centre de Ressources et Recherches Technologiques (C2RT), Institut Pasteur, Paris, France

${ }^{4}$ Hub Bioinformatique et Biostatistique, Département de Biologie Computationnelle - USR 3756 CNRS, Institut Pasteur, Paris, France.

${ }^{5}$ St Catharine's College Cambridge, UK, CB2 1RL

${ }^{6}$ Transcriptome \& Epigenome Platform - Biomics Pole - C2RT, Institut Pasteur Paris, France.

7 Immunologie des maladies infectieuses, Institut Pasteur de Madagascar, Antananarivo, Madagascar

${ }^{8}$ Unité de recherche sur le paludisme, Institut Pasteur de Madagascar, Antananarivo, Madagascar

${ }^{9}$ Unité d'entomologie médicale, Institut Pasteur de Madagascar, Antananarivo, Madagascar

${ }^{10} \mathrm{G} 4$ Malaria Group, Institut Pasteur de Madagascar, Antananarivo, Madagascar

\#a Current Address: Malaria Alert Centre, University of Malawi College of Medicine, Blantyre, Malawi.

\#b Current address: MRC Unit The Gambia at London School of Hygiene and Tropical Medicine, Fajara, Gambia

*Corresponding author: majoline.gusse81@gmail.com, catherine.bourgouin@pasteur.fr Author names and Email

Majoline Tchioffo Tsapi: majoline.gusse81@gmail.com

Etienne Kornobis: etienne.kornobis@pasteur.fr

Nicolas Puchot: nicolas.puchot@pasteur.fr 
Edward Solomon English: esae2@cantab.ac.uk

Caroline Proux: caroline.proux@pasteur.fr

Marie-Agnès Dillies: marie-agnes.dillies@pasteur.fr

Jessy Goupeyou-Youmsi: jessgoupez@gmail.com

Milijaona RANDRIANARIVELOJOSIA: milijaon@pasteur.mg

Romain Girod: rgirod@pasteur.mg

Anavaj Sakuntabhai: anavaj.sakuntabhai@pasteur.fr

Ousmane Ndiath: ousmane.ndiath@gmail.com

Catherine Bourgouin: catherine.bourgouin@pasteur.fr 


\begin{abstract}
Plasmodium vivax malaria is now recognized as the second most dangerous parasitic threat to human health with the regular decrease of Plasmodium falciparum worldwide over recent decades. A very limited numbers of studies address the interaction of $P$. vivax with its Anopheles mosquito vectors. Those studies were conducted in P. vivax endemic countries with P.vivax local major vectors for which limited genomic and genetic tools are available. Despite the presence of $P$. vivax in several African countries and increasing reports on its occurrence in many others, there is virtually no data on the molecular responses of Anopheles arabiensis, a major African mosquito vector, to $P$. vivax, which limits the development of further "mosquito-targeted" interventions aimed at reducing $P$. vivax transmission. Taking advantage of the situation of Madagascar where P. falciparum, P. vivax and An. arabiensis are present, we explore the molecular responses of $A n$. arabiensis towards these two human malaria parasites. RNA sequencing on RNAs isolated from mosquito midguts dissected at the early stage of infection (24 hours) was performed using mosquitoes fed on the blood of $P$. vivax and $P$. falciparum gametocyte carriers in a field station. From a de novo assembly of An. arabiensis midgut total RNA transcriptome, the comparative analysis revealed that a greater number of genes were differentially expressed in the mosquito midgut in response to $P$. vivax (209) than to $P$. falciparum (81). Among these, 15 common genes were identified to be significantly expressed in mosquito midgut 24 hours after ingesting $P$. vivax and P. falciparum gametocytes, including immune responsive genes and genes involved in aminoacid detoxification pathways. Importantly, working with both wild mosquitoes and field circulating parasites, our analysis revealed a strong mosquito genotype by parasite genotype interaction. Our study also identified 51 putative long non-coding RNAs differentially expressed in An. arabiensis mosquito infected midgut. Among these, several mapped to the published $A n$. arabiensis genome at genes coding immune responsive genes such as gambicin 1, leucine-rich repeat containing genes, either on sense or antisense strands.

This study constitutes the first comparison of An. arabiensis molecular interaction with $P$. vivax and $P$. falciparum, investigating both coding and long non-coding RNAs for the identification of potential transcripts, that could lead to the development of novel approaches to simultaneously block the transmission of vivax and falciparum malaria.
\end{abstract}




\section{Introduction}

Malaria is still a major public health concern in many tropical and subtropical countries (WHO 2020). Although malaria eradication has emerged as a goal for the next decade, the field consensus is that the development of novel intervention strategies is hindered by our still limited understanding of the biology of Plasmodium, the causative agent of malaria, and of the complex interactions that the parasite maintains with its mammalian and mosquito hosts.

With more than 2-decade of intense focus on Plasmodium falciparum, which is responsible for the majority of deaths, major progresses have been achieved leading to substantial falciparum malaria decrease, mostly outside Africa (WHO 2020). This happened despite the worrying appearance of artemisinin-resistant $P$. falciparum parasites in South-east Asia (Menard and Dondorp 2017). Plasmodium vivax is the most geographically widespread species among the human malaria parasites infecting an 14-25 million people annually (Howes, Battle, et al. 2016; Battle et al. 2019). Commonly considered as a mild disease, $P$. vivax malaria is responsible for numerous severe cases, the frequency of which is now clearly reported (Anstey et al. 2012; Baird 2013; Rahimi et al. 2014; Bourgard et al. 2018; Mukhtar et al. 2019).

Occurrence of $P$. vivax malaria is overall rare in Sub-Saharan Africa submerged by the deadly $P$. falciparum malaria (Battle et al. 2019; Weiss et al. 2019). It has classically been considered that $P$. vivax parasites were unable to infect individuals from black ancestry and further argued that absence of $P$. vivax in population from black ancestry was linked to the absence of the $P$. vivax receptor (the Duffy Antigen Receptor for Chemokines, DARC) on the red blood cells of those individual (Miller et al. 1978). In the few African countries with admixed populations of diverse genetic ancestry (Mauritania, Ethiopia and Madagascar), P. vivax is often the second cause of malaria (Solomon, Kahase, and Alemayehu 2020; Howes, Mioramalala, et al. 2016; Ba et al. 2016). In those countries, the same mosquito species do transmit both parasite species, with Anopheles arabiensis and its sister taxa Anopheles gambiae as major vectors mostly known as the major vectors of $P$. falciparum in sub-Saharan Africa.

Facing the absence of an efficient vaccine, increased resistance of malaria parasites to artemisininbased combined therapies (ACT) and increased resistance of mosquitoes to insecticides, transmission-blocking approaches have been placed at the forefront of additional strategies to combat malaria (Rabinovich et al. 2017). Transmission blocking (TB) strategies come in two 
"flavours": targeting the parasite stages developing into mosquitoes or targeting mosquito molecules essential for malaria parasite development into their vectors (Lavazec and Bourgouin 2008; Saul 2007). Recent advances in mosquito transgenesis mixing both parasite and mosquito targets have demonstrated that combined approaches might be feasible (Isaacs et al. 2012). Transmission blocking approaches based on mosquito molecules have almost exclusively focused on $P$. falciparum in the last decades with numerous studies of $P$. falciparum interaction with its major African vector An. gambiae, for which molecular and genetic tools have been developed. Today a limited number of targets has been proposed as target for transmission blocking strategies. Most of these targets correspond to mosquito molecules expressed in the mosquito midgut, the compartment where malaria parasites initiate their sporogonic development (Dinglasan et al. 2007; Lavazec et al. 2007; Zhang et al. 2015). Indeed, during the parasite early sporogonic development a limited number of ookinetes, the midgut invasive stage, are produced, representing a weak point for efficient intervention (Gouagna et al. 1998; Smith, Vega-Rodríguez, and Jacobs-Lorena 2014). Target validation is usually accomplished by experimental mosquito feeding on Plasmodiuminfected blood supplemented with antibodies raised against the candidate transmission- blocking molecule. As $P$. vivax is not cultivable yet, validation of TB candidates derived from An. gambiae$P$. falciparum studies were performed in P. vivax endemic countries and its local vectors. These resulted in contrasting results of modest robustness due to the regulatory difficulty to include $A n$. gambiae -P. falciparum as an internal control. Nevertheless, ingestion of antibodies against $A n$. gambiae APN1 and FRET1 reveals similar trends in reducing P. falciparum and P. vivax in An. gambiae and Anopheles dirus, respectively (Armistead et al. 2014; Niu et al. 2017). On the contrary, antibodies against AgSGU, an An. gambiae midgut GPI-anchored protein reduced development of $P$. falciparum in An. dirus but had no effect on $P$. vivax development in this vector (Mathias et al. 2014). This latter data is in line with previous ones that suggest that the development of P. falciparum and P. vivax in Anopheles tessellatus involves both common and different molecular mechanisms (Ramasamy et al. 1996).

With the advances of Next Generation Sequencing (NGS) technologies, transcriptomic approaches are being developed to gain further insights into Anopheles responses to P. vivax (Santana et al. 2019; Boonkaew et al. 2020; Kumari et al. 2021). Although providing substantial novel data, these studies were conducted with local Anopheles vectors for which limited molecular information or genetic tools are available and did not address the specificity of the mosquito response to either 
human Plasmodium species. Taking advantage of the situation of Madagascar where both $P$. falciparum and P. vivax are responsible for malaria and are transmitted mostly by An. arabiensis we performed a differential midgut transcriptomic analysis of local An. arabiensis mosquitoes infected with circulating strains of $P$. falciparum and $P$. vivax with the aim to identify molecules or pathways that could constitute targets for transmission-blocking approaches of either or both $P$. vivax and P. falciparum. At the initiation of the project, an RNA-Seq pilot study revealed a substantial sequence divergence of the Malagasy An. arabiensis RNA complement with the published An. arabiensis annotated genome that used a mosquito colony established from Sudan origin (VectorBase). Therefore, we chose to perform a de novo transcriptome assembly from our complete sets of RNA-Seq data. Our differential analysis reveals that 209 genes were differentially expressed in An. arabiensis midgut cells in response to $P$. vivax whereas only 81 were found differentially expressed upon P. falciparum infection, among which several transcripts encoding lncRNAs. A limited number of genes were regulated by both parasite species, which might facilitate selection of targets for limiting Plasmodium transmission.

\section{Materials and Methods}

\section{Study area and mosquito production}

The study took place in a field station established in Andriba (17³5'49.92" S, 46 56'0.59" E), Maevatanana district in the Northwest of Madagascar (Goupeyou-Youmsi et al. 2020). In this area, malaria is endemic and mainly due to $P$. falciparum and $P$. vivax, with $P$. falciparum being the most prevalent parasite species. The highest intensity of transmission is observed from November to April (Nguyen et al. 2020). Major and secondary Malagasy malaria vectors are present in the area: An. gambiae, An. arabiensis, Anopheles funestus, Anopheles mascarensis and Anopheles coustani. Anopheline larvae and pupae were collected daily in rice fields and water ponds in the Andriba village, using the standard dipping technique (Service 1993). After removal of nonanopheline larvae and predators, larvae and pupae were placed in clean water and larvae fed on Tetramin Baby and grounded cat-food until adult emergence. Morphological identification of adult mosquitoes revealed that over $95 \%$ of the collected larvae and pupae were indeed A. gambiae s.l. which includes both An. gambiae s.s and An. arabiensis, two morphologically indistinguishable sibling species. Only those adults were maintained in $30 \times 30 \mathrm{~cm}$ cages with ad libidum access to a 
$10 \%$ sucrose solution, in the field insectary. Day-light conditions of the insectary matched the natural local day-night cycle close to $12: 12$. Humidity was controlled by regular floor watering. In addition, adult cages were covered with wet tissue and placed behind plastic curtains to limit dryness and too high temperatures. Day-time temperature in the insectary matched roughly the local temperature while night-time temperature decreased by a few degrees only, by keeping the insectary doors tightly closed. Both temperature and humidity were recorded daily. Overall, temperature ranged from 24 to $28^{\circ} \mathrm{C}$ and humidity close to $80 \%$.

\section{Recruitment of Plasmodium vivax and Plasmodium falciparum gametocyte carriers}

Recruitment of $P$. vivax gametocyte carriers was performed among symptomatic patients attending the health centers of Antanimbary (17¹1'06.6"S 4651'19.4"E) and Andriba (17³5'49.92" S, $46^{\circ} 56^{\prime} 0.59^{\prime}$ ' E). Indeed, P. vivax gametocytes develop at any red-blood cell invasion cycle and are therefore, found in symptomatic individuals. On the contrary, P. falciparum mature gametocytes need 10 to 15 days to develop as an escape strategy away from the initial disease symptoms. Therefore, P. falciparum gametocyte carriers were selected among asymptomatic 5- to 14-yearold children who attended the local primary schools. For each child, a blood sample obtained by finger-prick was used to perform a RDT (malaria Pf/pan SD Bioline), thick and thin blood smears. The RDTs allow detection of $P$. falciparum and any of the others Plasmodium parasites, $P$. vivax, Plasmodium ovale and Plasmodium malariae, which all are present in Madagascar. Microscopy analysis was used to determine Plasmodium species and parasites stages. Asexual (trophozoïtes) and sexual (gametocytes) stages of $P$. vivax and $P$. falciparum were detected by light microscopy (100X magnification) on 10\% Giemsa-stained thick blood smears. Plasmodium gametocyte density was determined against 500 white blood cells (WBC), assuming the standard number of $8,000 \mathrm{WBC} / \mu \mathrm{l}$ of blood, using the $10 \%$ Giemsa-stained thin blood smears. An Artemisinin-based combination therapy (ACT) was given to each child with a positive RDT or carrying Plasmodium trophozoïtes, as evidenced by microscopy, according to the national guidelines. Children identified as $P$. vivax or $P$. falciparum gametocyte carriers were enrolled for providing blood for mosquito infections after their parents or legal guardians had signed documents indicating informed consent. All procedures involving human subjects used in this study were approved by the national ethical committee of the Malagasy Ministry of Health ( $\left.\mathrm{N}^{\circ} 122-\mathrm{MSANT} / \mathrm{CE}\right)$. 


\section{Experimental design}

Our global objective was to compare the mosquito midgut response to $P$. vivax and to $P$. falciparum. Because we worked with circulating Plasmodium parasites, Plasmodium isolates used for replicate infection experiments are likely different from each other. To be able to study mosquito midgut transcriptome regulation in response to the developing parasites, our experimental strategy requires performing two concurrent mosquito infections for each gametocyte sample (Figure S1). The first infection was performed with "infective" gametocytes and the second with heat inactivated gametocytes, there after termed "non-infective" gametocytes. In addition, as we worked with F0 from wild mosquitoes that likely have different genetic backgrounds, we aimed to consider individual midgut samples as mosquito biological replicates per gametocyte sample. Further, to avoid technical bias, individual mosquito midguts were selected from 2 (P. vivax) and 3 (P. falciparum) technical replicates per gametocyte sample. Overall, we selected 4 independent infections for each parasite species that resulted in prevalence of infection close to or above $40 \%$ as determined by oocyst detection in the "infective sample" 7 days post infection. After RNAs isolation and quality control 16 individual midguts for P. vivax and 24 individual midguts for $P$. falciparum were processed for RNA-Seq.

\section{Mosquito experimental infections}

Batches of 50 to 70 females 3 to 5 days-old were dispatched in homemade feeding pots and starved from sugar 16 hours before infection. From selected Plasmodium gametocyte carriers $5 \mathrm{ml}$ of venous blood were drawn in heparinized tubes. The blood sample was centrifuged at 2,000 rpm, 3 min and $37^{\circ} \mathrm{C}$ and the patient serum was replaced with $\mathrm{AB}$ serum from donors never exposed to malaria (EFS-France) to limit possible effect of acquired transmission-blocking immunity (Boudin et al. 2005). The blood sample was divided into two parts. The first part was directly proposed to mosquitoes and constitutes the "infective gametocyte sample"; the second part was heated and shaken at $42{ }^{\circ} \mathrm{C}$ for 20 minutes (Eppendorf Thermomixer Comfort) to inactivate Plasmodium gametocytes before being proposed to mosquitoes. This constitutes the "non-infective gametocyte sample" (Figure S1). Heat treatment was previously demonstrated to fully inhibit gametocyte infectivity (Mendes et al. 2008).

Membrane feeding assay was carried out essentially as previously described (Tchuinkam et al. 1993), using stretched Parafilm ${ }^{\circledR}$ and glass feeders maintained at $37^{\circ} \mathrm{C}$. Female mosquitoes were 
allowed to feed for $60 \mathrm{~min}$, in the dark. Fully fed females were transferred into small cages, matching each experimental pot, and given free access to a $10 \%$ sucrose solution. At 24 hours post blood meal (PBM), individual midguts were dissected in cold 1X PBS, transferred into $1.5 \mathrm{ml}$ micro-tube containing $100 \mu \mathrm{l}$ of Tri-Reagent (MRC) and stored at $-20^{\circ} \mathrm{C}$ for few days before being stored at $-80^{\circ} \mathrm{C}$ at the Institut Pasteur de Madagascar. Carcasses from each dissected mosquito were individually stored in micro-tubes for further species identification. The remaining mosquitoes were maintained under optimal conditions in the insectary and their midgut dissected on day $7 \mathrm{PBM}$ to determine prevalence and load of infection by counting oocysts on mercurochrome $(0.4 \% \mathrm{w} / \mathrm{v}$ - Sigma-Aldrich-M7011) stained midguts using a light microscope (X20 \& X40).

At the end of the transmission season, all biological samples were sent under dry ice to Institut Pasteur in Paris for RNA-Seq analysis.

\section{Mosquito species identification}

Although we had previous indication that the majority of the A. gambiae s.l. present in Andriba belongs to the An. arabiensis taxon, we used a PCR based procedure to confirm that indeed all infected samples were from An. arabiensis mosquitoes. For that, DNA was extracted from each mosquito carcass matching the prepared midgut samples, using DNAzol® reagent (MRC). PCR primers were as previously published (Fanello, Santolamazza, and della Torre 2002) (Table S3). Mosquitoes from our An. gambiae Yaoundé colony and a recently established An. arabiensis colony were used as control.

\section{RNA extraction and reverse transcription}

Individual mosquito midguts stored in $100 \mu 1$ Tri-Reagent were crushed using polypropylene micro-pestles (Dutscher 045007). Total RNA purification was performed using the Direct-zol ${ }^{\mathrm{TM}}$ RNA Microprep isolation kit (Zymo Research, Cat \# R2062), according to the manufacturer's instructions. The Tri-Reagent extract was loaded onto Microprep column and total RNA was eluted with $10 \mu 1$ of DNase/RNAse free water and further treated with DNAse (Invitrogen ${ }^{\mathrm{TM}}$ TURBO DNA-free ${ }^{\mathrm{TM}} \mathrm{Kit}$ ). RNA quantification was performed using Nanodrop One (Thermo Scientific). 
Before initiating the RNA-Seq experiments, we verified that every single "infected" midgut contained developing Plasmodium parasites by detecting the ookinete mRNA marker encoding Pvs25 or Pfs25 using primer sets reported by (Wampfler et al. 2013) and q-PCR (Power SYBR ${ }^{\mathrm{TM}}$ Green PCR Master Mix amplification - Life Technologies).

Reverse transcription reactions were performed in $40 \mu 1$ final volume using $3 \mu 1$ of RNA, $8 \mu 1$ of $5 \mathrm{X}$ First-Strand Buffer, $4 \mu \mathrm{l} 0.1 \mathrm{M}$ DTT, $1 \mu \mathrm{l}$ of $20 \mathrm{mM}$ dNTPs, $2 \mu 1$ of $50 \mu \mathrm{M}$ random hexamers, $1 \mu 1$ of $40 \mathrm{U} / \mu 1 \mathrm{RNaseOUT}{ }^{\mathrm{TM}}$ recombinant Ribonuclease Inhibitor and $2 \mu 1$ of 200 Units MMLV Reverse Transcriptase. All reagents were from Life Technologies Invitrogen, except Random hexamers obtained from Promega.

\section{RNA-Seq library preparation and Illumina sequencing}

RNA quality was assessed using the Agilent Bioanalyzer. Briefly, selected RNA samples were denatured, by incubating at $70^{\circ} \mathrm{C}$ for 2 minutes, and then placed on ice. Nine $\mu l$ of the gel-dye mix was pipetted into the bottom of a nanochip. Then, $1 \mu 1$ of samples, $1 \mu 1$ of RNA 6000 Nano Marker and $1 \mu 1$ of RNA 6000 Ladder were pipetted into assigned well, vortexed and run using 2100 expert Eukaryote Total RNA Nano Series II (Agilent Technologies).

Forty RNA samples of good quality obtained from infected (infective gametocytes) and cognate uninfected (heat-inactivated gametocytes) individual mosquito midguts were selected for cDNA library preparation: 16 corresponding to $P$. vivax and 24 to $P$. falciparum (Figure S1). RNA-Seq library preparation was performed using the SMARTer ${ }^{\circledR}$ Stranded Total RNA-Seq kit - Pico Input Mammalian (Takara Bio USA, Inc) with the RNA input ranging from 7 to $10 \mathrm{ng}$ of starting material.

The protocol starts with first-strand cDNA synthesis from total RNAs fragmented for 3 minutes following Takara's recommendation. The Illumina adapters and indexes were added, followed by the purification of the RNA-Seq library and depletion of ribosomal cDNA with ZapR and RProbes. The final RNA-Seq library was obtained after 15 cycles of PCR amplification and purified using Agencourt AMPure XP beads (Beckman and Coulter, Beverly, MA, USA). The library size distribution was evaluated by running samples on the Agilent 2100 Bioanalyzer. The 40 libraries were then sequenced using the HiSeq 2500 (Illumina, San Diego, CA, USA) at 2x100 bp pairedend sequencing with v4 chemistry. The 40 samples were distributed on the 8 lanes of the flow cell such that there was no confounding effect between the lanes and the biological factors of interest. 
Library preparation and sequencing were performed at the Institut Pasteur Epigenetics and Transcriptomics Platform (PF2).

\section{de novo assembly of $\mathbf{A n}$. arabiensis midgut transcriptome}

From a pilot study using STAR 2.7.0d and the available assembly of the An. arabiensis genome on VectorBase, only $25 \%$ of the RNA-Seq reads from our Malagasy An. arabiensis samples could be mapped. In addition, functional annotation was scarce. Therefore, we performed a de novo midgut transcriptome assembly using the paired-end sequencing.

Quality control benefited from MultiQC 1.7 (Ewels et al. 2016). The resulting reads (30-90 million) displayed in Table S2 were quality trimmed and adapters clipped using fastp 0.19.6 (Chen et al. 2018) prior to de novo assembly using Trinity 2.6.6 (Grabherr et al. 2011). Completeness of the transcriptome was evaluated by BUSCO 3.0.2 (Simão et al. 2015), comparing with Diptera and Insecta reference transcriptomes.

\section{Functional annotation and differential expression analysis}

Functional annotation of the midgut transcriptome was performed with Trinotate 3.1.1 (Bryant et al. 2017) which uses Blast 2.5.0 (Camacho et al. 2009), HMMER 3.2 (Finn, Clements, and Eddy 2011), TMHMM 2.0 (Krogh et al. 2001), SignalP 4.0 (Petersen et al. 2011) and RNAmmer 1.2 (Lagesen et al. 2007). Two groups of transcribed features were obtained: annotated features originating from known coding genes and unannotated features that could correspond to previously undescribed genes or non-coding RNA. The PLEK 1.2 (Li, Zhang, and Zhou 2014) and CPAT 1.2.4 (Wang et al. 2013) non-coding RNA identification programs were next used to refine the unannotated features. In order to investigate further their function, putative lncRNA transcripts were mapped to An. arabiensis Dongola AaraD1 genome and its annotation AaraD1.11 to identify potential target genes.

Transcript expression levels comparing infected versus uninfected An. arabiensis mosquitoes were quantified using kallisto 0.45.0 (Bray et al. 2016). Transcript abundances were used for gene-level comparisons using tximport 1.6.0 (Soneson, Love, and Robinson 2015) from the three and four biological replicates of $P$. vivax infection and P. falciparum infection. DESeq2 1.18.1 (Love, Huber, and Anders 2014) was used to detect genes differentially expressed between the infected and uninfected mosquito midgut samples. Differentially expressed genes were further analyzed for 
functional classification using Gene Ontology (GO). GO enrichment analysis was performed using Trinity scripts and GOSeq 1.30 .0 (Young et al. 2010) to provide a shortlist for potential genes of interest classified in three categories: biological process, molecular function and cellular component.

\section{Code and data availability}

The bioinformatic analysis will be provide later as Jupyter notebooks. The RNA-Seq reads, metadata and raw reads count will be available after submission of the manuscript for publication. Differential expressed genes and GO term analysis for each Plasmodium isolate are also presented in Supplementary Tables 4 to 6 .

\section{Results}

\section{Selection of mosquito midgut samples for the differential RNA-Seq analysis}

From $14 \mathrm{An}$. arabiensis experimental infections performed in our field station between January and March 2017, we selected 4 infections with $P$. vivax and 4 infections with $P$. falciparum (Table $\mathrm{S} 1$ and Figure S2). The inclusion criteria were enough individual dissected mosquito midguts $24 \mathrm{~h}$ post blood meal, prevalence of infection at day 7 post infection in the experimental "infective" blood meal close to or above $40 \%$ and absence of parasites in the "non-infective" blood meal at day 7 post infection. Although $P v 3$ exhibited a reduced prevalence of infection at $\mathrm{d} 7(17.5 \%)$ it was initially included in our analysis. The second criterion was detection of the mRNA encoding the Plasmodium ookinete surface protein 25 (Pvs25 or Pfs25) in individual midguts by RT-qPCR. The last criterion was the quality of the RNA extracts for subsequent RNA-Seq library construction as determined using the Agilent Bioanalyzer. Overall, as depicted in Figure S1, 16 RNA-Seq libraries were produced from mosquitoes challenged with $P$. vivax and 24 from mosquitoes challenged with $P$. falciparum.

\section{Summary of RNA-Seq raw data}

Sequencing of the 40 libraries resulted in a total of 1.3 billion paired-end reads. From the pairedend reads, de novo midgut An. arabiensis transcriptome assembly was carried out and produced 
250642 transcripts; 112242 of them being supported by at least 5 reads and correspond to 164954 putative genes (i.e. trinity clusters). Completeness of the transcriptome was evaluated at $78 \%$ and 94\% by BUSCO, respectively comparing with Diptera and Insecta reference transcriptomes. Annotation performed by the Trinotate suite led to 60243 transcripts showing significative similarity with swissProt or Pfam entries, 12083 having a blastx hit with Drosophila melanogaster nucleotide sequence. From the transcripts, we performed a Principal Component Analysis (PCA) based on VST (variance stabilizing transformation) transformed counts from DESeq2 to visualize the overall effect of the infected and the uninfected status of the mosquito midguts according to each Plasmodium species and isolate (Figure S3). We excluded from the PCA, Pv3 samples as the corresponding libraries had much less sequencing coverage than the others. Considering that the mosquito samples originated from different field collections across the malaria transmission season and that the parasite isolates came from different gametocyte carriers, it was anticipated that significant genetic variation could be observed among mosquito samples and among parasite isolates. The PCA shows that components 1 and 2 together display $58 \%$ and $52 \%$ of the total variance in response to $P$. vivax and $P$. falciparum infection, respectively.

Three clusters were clearly identified representing the three independent $P$. vivax mosquito infections (Figure S3A). Nonetheless, no clear structure was observed corresponding to the infected/uninfected status of the mosquitoes. The results were slightly different with $P$. falciparum with a trend of clustering between infected and uninfected mosquitoes within a $P f$ isolate (Figure S3B). More striking was the clustering of $P f 1$ and $P f 2$ data. Indeed, $P f 1$ and $P f 2$ infections used the same batch of mosquitoes, suggesting a strong contribution of mosquito genetic component. Based on this PCA analysis, and since there was extensive variation within groups and no clear overall segregation between infected and uninfected mosquito midguts, we performed individual differential analyses using each parasite isolate separately $(P v 1, P v 2, P v 4, P f 1, P f 2, P f 3$ and $P f 4)$ comparing infected versus uninfected mosquito samples.

\section{Differential gene expression in An. arabiensis midguts infected with $P$. vivax}

Using a False Discovery Rate of 0.05 (FDR < 0.05) as the threshold to classify differentially expressed genes, our analysis resulted in the identification of 209 transcripts differentially regulated in An. arabiensis midguts $24 \mathrm{~h}$ after feeding on infective $P$. vivax gametocytes compared to mosquito fed on cognate un-infective gametocytes. The detailed gene list is provided in Table 
S4. From the 209 transcripts identified in mosquito midguts as differentially expressed in response to $P$. vivax isolates, 36 were up-regulated and 173 genes were down-regulated (Figure 1A). The majority of the transcripts were specific to each $P$. vivax isolate. Five down-regulated transcripts were shared between $P$. vivax isolates 1 and 4 (Figure 1B and Table 1). The most down-regulated An. arabiensis gene was TRINITY_DN109598_c5_g1 with two transcripts showing a $\log _{2} \mathrm{FC}$ at 6,357 identified in $P v 1$ infection. This gene showed 36.89\% identity to Aminopeptidase N_bovin. Another down regulated transcript, TRINITY_DN108067_c0_g1 in Pv2 infection showed 98.04\% identity to An. gambiae Defensin 1 with $\log _{2} \mathrm{FC}$ at -4.13 . The most up-regulated transcripts were TRINITY_DN101897_c5_g2 with three variants showing $\log _{2} \mathrm{FC}$ at 4.549 identified in Pv4 infection, and TRINITY_DN103245_c1_g1 showing $\log _{2} \mathrm{FC}$ at 4.551 in Pv1 infection; both transcripts were classified as unannotated genes.

\section{Differential gene expression in $\boldsymbol{A n}$. arabiensis midguts infected with $\boldsymbol{P}$. falciparum}

Eighty-one (81) transcripts were identified differentially regulated in An. arabiensis midguts in response to $P$. falciparum, 36 were up-regulated and 45 were down-regulated (Figure 2A,B and Table 1). Surprisingly, there was no common transcript between the four infections with $P$. falciparum isolates. The most down-regulated An. arabiensis gene was TRINITY_DN115268_c5_g1 in Pf1 infection showing a $\log _{2} \mathrm{FC}$ at -4,154. This gene showed 34.11\% identity to Membrane alanyl aminopeptidase (AMPM_HELVI) from Heliothis virescens. The second most down-regulated transcript, TRINITY_DN102799_c3_g1 in Pf3 infection, had a $\log _{2} \mathrm{FC}$ at $-3,624$. This gene exhibits $69.24 \%$ identity to Cytochrome P450 from Drosophila. The most up-regulated transcript was TRINITY_DN114835_c4_g2 g, in Pf3 infection with a $\log _{2} \mathrm{FC}$ at 4,113 and correspond to an unannotated gene.

\section{Differential expression of long non-coding RNAs in An. arabiensis infected with Plasmodium}

Among the 290 genes differentially expressed transcripts in response to either $P$. vivax or $P$. falciparum, 51 genes were classified as unannotated features. Using non-coding RNA identification programs, these 51 features were identified to be putative lncRNAs representing a total of 146 transcripts assembled by trinity. Among the 51 lncRNA genes, 14 were upregulated and 23 downregulated associated to $P$. vivax infection whereas 11 lncRNAs were upregulated and 4 downregulated associated to $P$. falciparum infection. No putative lncRNA was found in 
transcripts from the Pf4 isolate. Among the upregulated lncRNAs, one was common to $A n$. arabiensis infected with $P v 4$ isolate and Pf3 isolate. An overview of lncRNAs regulation is presented in Figure 2C and Table S5.

Of the 51 genes classified as unannotated features, 18 candidates were successfully mapped to the An. arabiensis Dongola genome at different loci. Three transcript features were identified as antisense lncRNAs (101849, 114154 and 106465) and are underlined in red in Table S5. They mapped respectively to Cuticular protein (TRINITY_DN101849_c1_g1 with five variants i1, i2, i3, i5 and i6 and showing $\log 2 \mathrm{FC}$ at -4,25), Phosphoserine phosphatase (TRINITY_DN114154_c2_g1 with five variants i1 to i5 showing $\log 2 \mathrm{FC}$ at -3.094) and Gambicin 1 (TRINITY_DN106465_c1_g3 with two variants i1 and i2 showing log2FC at -2.78). The 15 others were identified as sense lncRNA transcripts, some of them being associated to mosquito immune responsive genes. These included, Leucine-rich immune protein (Long) (TRINITY_DN112064_c0_g5_i1 with $\log 2 \mathrm{FC}$ at -2.94), peptidoglycan recognition protein (TRINITY_DN107658_c0_g1_i1 with $\log 2 \mathrm{FC}$ at -3.29), leucine-rich repeat protein (TRINITY_DN110940_c0_g1 with four variants i1, i5, i4 and i6 showing log2FC at 2.52) and Protein Kinase cGMP-dependent (TRINITY_DN113382_c1_g2 with four variants i1 to i4 showing $\log 2 \mathrm{FC}$ at 2.38). A schematic alignment of the lncRNAs matching mosquito immune responsive genes is presented in Figure 3.

\section{An. arabiensis midgut transcripts regulated by both $P$. vivax and $P$. falciparum}

As presented above, there was a clear mosquito genotype to pathogen genotype interaction within parasite species. Our initial objective was to identify mosquito genes regulated similarly by both parasite as well as genes differently regulated by $P$. vivax and $P$. falciparum. We therefore looked in more details to An. arabiensis response to both human parasites, keeping in mind that the mosquito genotype (multiple F0 individuals) was not fixed. We identified 3 classes of transcripts: 1) those that were down regulated in both $P$. vivax and $P$. falciparum infected mosquitoes, 2) those that were upregulated in both groups, 3) those that were down regulated in $P$. vivax infected mosquitoes and upregulated in P. falciparum infected mosquitoes. No transcript upregulated in $P$. vivax infected mosquitoes and downregulated in P. falciparum infected mosquitoes was detected. The Venn diagram presented in Figure 1C highlighted the impact of parasite by mosquito genotype interaction for classes 1 and 2. Indeed, 3 transcripts were found down-regulated in both $P v 1$ and 
$P f 3$ infected mosquitoes, 8 transcripts down-regulated in $P v 4$ and $P f 3$ infected mosquitoes and an additional 1 down-regulated in $P v 1$ and $P f 4$, whereas only one commonly up regulated transcript was shared between $P v 4$ and Pf3. From these data hierarchical clustering and heat maps were generated (Figure 4). In the heat maps, 11 class1 transcripts are globally downregulated in both $P$. vivax and $P$. falciparum infected mosquitoes (pink square) although data from two Pf4 midguts and one Pv2 midgut depart from the global trend. One transcript belonging to class 2 correspond to an unannotated gene (TRINITY_DN113404) further identified as a lncRNA (Table S5). Class 3 included three genes: Defensin1 (Def1), Peroxiredoxin-1,6 (PRDX6/PRX1), and Clavesin 1 (CLVS1). The overall differential regulation between $P v$ and $P f$ infected mosquitoes is nevertheless modest, with slight variation according to the parasite genotype. While Defensin1 and Peroxiredoxin have been associated to Plasmodium-mosquito interaction, the detection of a Clavesin related transcript is quite elusive in Anopheles-Plasmodium interaction as this gene family has been described as encoding Neuron-specific Lipid- and Clathrin-binding Sec14 Proteins (Katoh et al. 2009).

\section{Gene Ontology classification of $\boldsymbol{A n}$. arabiensis genes regulated upon Plasmodium infection}

Differentially expressed genes were further analyzed for functional classification using Gene Ontology (GO). GO enrichment analysis was performed using Trinity scripts and GOSeq 1.30.0 (Young et al. 2010) to provide a shortlist for potential genes of interest classified in three categories: biological process (BP), molecular function (MF) and cellular component (CC).

A summary of detailed annotations for each category corresponding to transcripts regulated in mosquito midgut upon each Plasmodium infection is depicted in Figure 5 and Table S6, using the highest number of enriched or depleted GO terms from Pf3 and Pf1 (Figure 5A) Pv1 and Pv4 (Figures 5B-D).

Metabolic and cellular processes were the most affected GO terms for biological process in mosquito midguts in response to $P$. falciparum and $P$. vivax infections: metabolic and biosynthetic processes for $P$. falciparum while proteolysis, multicellular organismal process, and developmental process for P. vivax (Figure 5A,B). The most changed GO terms for molecular function in mosquito midgut were binding activity in response to P. falciparum (Figure 5A) and mainly catalytic activity, peptidase and endopeptidase activity for P. vivax infections (Figure 5C). 
For the cellular component category, the enrichment analysis revealed the strongest enrichment of transcripts associated to cellular_component for both parasite species (Figures 5A and 5D).

\section{Discussion}

We present in this report the first comparative investigation on An. arabiensis response to the two most important human malaria parasites $P$. falciparum and $P$. vivax using an RNA-Seq approach. Our study focused on RNAs differentially expressed in the mosquito midgut $24 \mathrm{~h}$ post ingestion of infective $P$. falciparum and $P$. vivax gametocytes. This temporal and tissue specific window correspond to the migration of the parasite ookinetes across the mosquito midgut which correspond to the most vulnerable stage of the parasite both in numbers (Gouagna et al. 1998) and their accessibility for transmission blocking interventions (Saul 2007; Lavazec and Bourgouin 2008; Duffy 2021).

Working in a field station with natural populations of both An. arabiensis and Plasmodium, our RNA-Seq clearly demonstrates the importance of mosquito genotype to parasite genotype interaction in the building up of the mosquito transcriptional response. Indeed, each set of RNASeq data segregated according to the mosquito sample (Figure S3) except for $P f 1$ and $P f 2$ samples, that correspond to the same mosquito field population collected over the same week. Influence of parasite genotype is evidenced when looking at the number of differentially regulated transcripts in $P f 1$ and $P f 2$ infected mosquitoes, where $P f 1$ led to 20 regulated transcripts between infected and uninfected mosquitoes whereas $P f 2$ led to only 4 regulated transcripts (Table 1), despite similar gametocytemia in the two isolates (Table S1). Similarly, influence of parasite genotype can be seen within a Plasmodium species. Indeed, only 5 differentially regulated transcripts between infected and uninfected mosquitoes were common to the $3 P v$ isolates, while none were found between the $P f$ isolates (Table 1).

Looking at the global response of An. arabiensis midgut tissue to $P$. vivax and $P$. falciparum infective gametocytes, our analysis reveals that wild Plasmodium isolates trigger the regulated expression of a limited number of transcripts, whether coding or not coding. Overall infection of An. arabiensis with $P$. vivax gametocytes regulate the expression of 209 transcripts while only 81 transcripts were regulated by P. falciparum gametocyte containing field isolates. The P.falciparum 
regulated transcript numbers are low compared to the higher numbers of regulated transcripts reported in transcriptomic studies of An. gambiae interaction with P. falciparum (Dong et al. 2006; Mead et al. 2012). However, these studies used established mosquito colonies (G3 or Keele strains) and referenced P. falciparum gametocyte producing strains (NF54 isolate or its 3D7 derivative clone). On the contrary, our data with $P$. vivax infected mosquitoes is in good agreement with results obtained with A. aquasalis (Santana et al. 2019) and An. dirus (Boonkaew et al. 2020), despite these studies used well established mosquito colonies. While the An. dirus study investigated whole mosquito transcriptome, revealing 313 regulated transcripts comparing mosquitoes fed on P. vivax gametocyte containing blood with mosquitoes fed on non -infected blood, the An. aquasalis study investigated mosquito midgut transcripts, revealing 49 regulated transcripts comparing mosquitoes fed on $P$. vivax gametocyte containing blood with mosquitoes fed on inactivated gametocytes containing blood, similar to our experimental design. A globally limited number of midgut transcripts was also reported in Aedes aegypti upon challenge with Dengue virus (Raquin et al. 2017). Interestingly, or intriguingly, our results, in contrast to the $A n$. aquasalis study, show that $P$. vivax down regulated a more significantly larger numbers of $A n$. arabiensis transcripts than the number of up regulated ones. This could suggest that in a fully natural field combination P. vivax manipulates its host for the sake of its transmission.

Among the commonly down regulated genes, three genes belong to the catabolism of amino acid and notably tyrosine, which is known to be toxic in blood feeding arthropods when in excess as a result of the blood digestion (Sterkel et al. 2016). The observation that both Plasmodium species down regulated the expression of mosquito genes encoding amino acid degradation pathway is suggestive of parasite manipulation of the host for its own benefit. By contrast, it is surprising that both parasite species were down regulating a mosquito transcript encoding a purine nucleoside phosphorylase (PNP), involved in purine metabolism, owing that Plasmodium parasites are purine auxotrophic (in (Taylor et al. 2007). As intriguing is the recent report that Caenorhabditis elegans PNP ortholog is a negative regulator of the worm response to pathogens (Tecle et al. 2021). Two genes involved in mosquito immune response (encoding a P450 and a serpin) were as well down regulated. Such regulation is compatible with a pathogen evasion strategy to the mosquito immune response. Interestingly, Plasmodium parasites were also down regulating two genes encoding cuticular component (CU17 and a CUD4 family cuticular glycoprotein), in line with the needs for 
Plasmodium ookinetes to traverse the mosquito midgut peritrophic matrix before reaching the midgut epithelial cell (Sieber et al. 1991; Shahabuddin et al. 1994). Lastly is might seem odd that two neurogenic Notch encoding genes were down regulated in Plasmodium infected mosquito midgut. However, recent work revealed that the Notch-Delta signaling pathway is involved in $A e$. aegypti response to DENV mosquito midgut infection (Serrato-Salas et al. 2018).

Our study led to the identification of several regulated transcripts corresponding to lncRNAs with only one common to $P$. vivax and $P$. falciparum infection. No match to annotated genes could be found for this upregulated lncRNA. Interestingly, among the lncRNAs four exhibit matches to immune responsive genes one being antisense to the An. arabiensis ortholog of Gambicin 1.

In conclusion, our comparative study of $P$. vivax and $P$. falciparum interaction with An. arabiensis is paving the way for a better understanding of the specific interaction of these malaria species with its anopheline vectors. Importantly, our study working with wild mosquitoes and wild parasites revealed a strong effect of mosquito by pathogen genotype interaction, which was not highlighted in any previous Plasmodium-Anopheles interaction studies. Our study highlights also for the first time, the regulation of lncRNAs, the function of which has yet to be determined. Additional functional analyses are required for selecting relevant genes as potential targets for $P$. vivax and P. falciparum transmission blocking approaches.

\section{Acknowledgments}

We are grateful to volunteers from Andriba primary schools and their parents or guardians for participating in this study. We also address warm thanks to the medical teams of the Andriba and Antanimbary basic health center (CSB) for their assistance in collecting blood samples, notably Dr Richard and Odja, Andriba midwife. Many thanks to the whole local population who supported our study: Andriba city major, school directors, Funkuntany delegates, local military authorities, and health district authorities based in Maevatanana. A specific thanks to Jean-Pierre, guardian of the Andriba health center and his constant help for field mosquito larvae collections. We are also grateful to the technician staff from the Institut Pasteur de Madagascar: Tianasao from the malaria 
Unit, Manda, Fidelis and Miara from the medical entomology Unit, not forgetting our drivers and cooks, René, Bari and Hery.

\section{Author Contributions}

Conceived the project and funding: CB. Designed the experiments: MTT, CB, M-AD. Provide local support: RG, MJ, AS, Performed the experiments: MTT, NP, CB, JMGY, SE, CP, ON. Analyzed the data: MTT, EK, CB. Contributed reagents/materials /analysis tools: CB. Draft the paper: MTT, EK, CB.

Funding: This work was supported by funds from the LabEx IBEID (The Laboratory of Excellence ( $L a b E x$ ) Integrative Biology of Emerging Infectious Diseases) to CB. The funders had no role in study design, data collection and analysis, decision to publish, or preparation of the manuscript.

\section{Figure Legend}

Figure 1. Volcano plots and numbers of An. arabiensis midgut differentially expressed genes upon $\boldsymbol{P}$. vivax challenge. A) Plots of each pairwise comparison between infected and uninfected mosquito midgut samples. Transcripts were considered differentially expressed with a $\log _{2}$-fold change $\geq 1$ and a corrected $p$-value (padj) $<0.05$. Red dots display up-regulated genes and blue dots down-regulated genes. B and C) Venn diagrams highlighting shared up and down regulated mosquito genes among individual $P$. vivax infections (B), and among $P$. vivax and $P$. falciparum individual infections (C). Upregulated genes are labelled in red and downregulated ones in black.

Figure 2. Volcano plots and numbers of An. arabiensis midgut differentially expressed genes upon $\boldsymbol{P}$. falciparum challenge. A) Plots of each pairwise comparison between infected and uninfected mosquito midgut samples. Transcripts were considered differentially expressed with a $\log _{2}$-fold change $\geq 1$ and a corrected $p$-value (padj) $<0.05$. Red dots display up-regulated genes and blue dots down-regulated genes. B) Venn diagram highlighting shared up and down regulated mosquito genes among individual $P$. falciparum infections. C) Venn diagram highlighting shared 
up and down regulated mosquito lncRNAs among individual $P$. vivax and $P$. falciparum infections. Upregulated genes are in red and downregulated genes in black.

Figure 3. Overlapping of An. arabiensis regulated IncRNAs with known Anopheles immune responsive genes. ncRNA transcripts were aligned to An. arabiensis genes extracted from the Dongola AaraD1 genome and its annotation AaraD1.11.

Figure 4. Heat map representation of shared differentially regulated genes in $\mathbf{A n}$. arabiensis mosquito midguts upon Plasmodium infection. A) P. vivax infection. B) P. falciparum infection. At the top of each heat map (A \& B) pink color represents infected samples, while green color represents non infected samples (ie mosquitoes fed on inactivated parasites, see M\&M). On panel A each P. vivax isolates (Pv1, Pv2 and Pv4) are color coded. Similarly, on panel B, each $P$. falciparum isolates (Pf1, Pf2, Pf3 and Pf4) are color coded. Each column is the measurement of change in gene expression (blue low expression and red high expression). Hierarchical clustering is represented by dendrogram trees. The pink rectangles delineate the 11 down-regulated genes in response to both $P v$ and $P f$ infection (T230: tryptophan 2,3-dioxygenase; ECE: Endothelinconverting enzyme; CUD4: Endocuticle structural glycoprotein ABD-4; PNPH: Purine nucleoside phosphorylase; CU17: Larval cuticle protein LCP-17; NOTC3/NOTCH: Neurogenic locus notch homolog protein 3; SGP1: Serine protease inhibitor I/II; NOTCH/SNED1: Neurogenic locus Notch protein; C4G15/FACR1: Cytochrome P450 4g15; HPPD: 4-hydroxyphenylpyruvate dioxygenase; ATTY: Tyrosine aminotransferase. The unannotated gene corresponds to an upregulated lncRNA in response to both $P$. vivax4 and $P$. falciparum 3 infection. Three genes were down-regulated in response to $P v$ and up-regulated in response to $P f$ (PRDX6/PRX1: Peroxiredoxin; CLVS1: Clavesin-1; DEF1: Defensin1).

Figure 5. Gene ontology enrichment analysis of $\boldsymbol{A n}$. arabiensis genes 24 hours following $\boldsymbol{P}$. vivax and $\boldsymbol{P}$. falciparum infection. A false discovery rate of $0.05(\mathrm{FDR}<0,05)$ was used for generating these graphs. A) Number of genes per category upon P. falciparum infection with isolates 1 and 3: biological process (BP), cellular component (CC) and molecular function (MF). B-D) Percentage of genes upon $P$. vivax infection with $P v l$ and $P v 4$ isolates, according to biological process category (B), molecular function (C) and cellular component (D). 
Table 1. : Summary of $\boldsymbol{A n}$. arabiensis transcript numbers differentially expressed upon $\boldsymbol{P}$. vivax and $P$. falciparum infection.

\section{Supporting files}

Table S1 Plasmodium isolate characteristics and An. arabiensis infection rates

Table S2: RNA-Seq data summary: Statistics on sequenced reads before and after filtering by fasp and their kallisto pseudo-mapping percentage.

Table S3. Primer sequences.

Table S4. List of annotated and unannotated transcripts differentially expressed in An. arabiensis mosquito midgut in response to $P$. vivax and $P$. falciparum infection.

Table S5. List of An. arabiensis long non-coding RNAs differentially expressed in response to $P$. vivax and $P$. falciparum infection.

Table S6. Functional classification of An. arabiensis midgut transcripts using gene ontology analysis. The three categories are obtained upon $P v$ and $P f$ infection based on the FDR $<0.05$.

Figure S1. Experimental design for mosquito infection by DMFA (Direct Membrane Feeding Assay) and RNA-Seq library production. Three-to-five-day-old female mosquitoes were starved from sugar $16 \mathrm{~h}$ prior blood-feeding on infective and non-infective blood containing $P$. vivax or $P$. falciparum gametocytes from field isolates. Individual mosquito midgut 24 post-blood-feeding were dissected in cold PBS and immediately transferred in Tri-Reagent. Samples were stored at $20^{\circ} \mathrm{C}$ for 3 to 4 days in the field station, before being placed at $-80^{\circ} \mathrm{C}$ in Antananarivo and later fetched to Paris on dry ice. After total RNA extraction. cDNAs for RNA-Seq library were obtained from the selected RNAs and a total of 40 libraries submitted to sequencing.

Figure S2. P. vivax and $P$. falciparum oocyst loads in infected $A$ n. arabiensis. The data represent the intensity of infection for each of the isolates used in the RNA-Seq analysis. Oocysts were detected on day 7 post infection. Additional details are presented in the M\&M section and in Table $\mathrm{S} 1$. The left $\mathrm{Y}$ axis applies to all infections except $\mathrm{Pf3}$ for which the right $\mathrm{Y}$ axis is drawn.

Figure S3. Principal Components Analysis based on normalized counts from individual mosquito midguts infected with $\boldsymbol{P}$. vivax or $\boldsymbol{P}$. falciparum. (A) PCA on global RNA-Seq patterns for mosquito fed on P. vivax infected and uninfected blood, PCA 1 and 2 displayed 58\% of total variance; three clusters were clearly identified according to the $P v$ isolates. (B) PCA on global 
RNA-Seq patterns for mosquito fed on P. falciparum infected and uninfected blood. Both PCA axis displayed $52 \%$ of total variance; three clusters were identified and PCA did not dissociate Pf1 and Pf2.

\section{References}

Anstey, Nicholas M., Nicholas M. Douglas, Jeanne R. Poespoprodjo, and Ric N. Price. 2012. 'Chapter Three - Plasmodium vivax: Clinical Spectrum, Risk Factors and Pathogenesis.' in S. I. Hay, Ric Price and J. Kevin Baird (eds.), Advances in Parasitology (Academic Press).

Armistead, J. S., I. Morlais, D. K. Mathias, J. G. Jardim, J. Joy, A. Fridman, A. C. Finnefrock, A. Bagchi, M. Plebanski, D. G. Scorpio, T. S. Churcher, N. A. Borg, J. Sattabongkot, and R. R. Dinglasan. 2014. 'Antibodies to a single, conserved epitope in Anopheles APN1 inhibit universal transmission of Plasmodium falciparum and Plasmodium vivax malaria', Infect Immun, 82: 818-29.

Ba, Hampâté, Craig W. Duffy, Ambroise D. Ahouidi, Yacine Boubou Deh, Mamadou Yero Diallo, Abderahmane Tandia, and David J. Conway. 2016. 'Widespread distribution of Plasmodium vivax malaria in Mauritania on the interface of the Maghreb and West Africa', Malaria Journal, 15: 80.

Baird, J. K. 2013. 'Evidence and implications of mortality associated with acute Plasmodium vivax malaria', Clin Microbiol Rev, 26: 36-57.

Battle, Katherine E., Tim C. D. Lucas, Michele Nguyen, Rosalind E. Howes, Anita K. Nandi, Katherine A. Twohig, Daniel A. Pfeffer, Ewan Cameron, Puja C. Rao, Daniel Casey, Harry S. Gibson, Jennifer A. Rozier, Ursula Dalrymple, Suzanne H. Keddie, Emma L. Collins, Joseph R. Harris, Carlos A. Guerra, Michael P. Thorn, Donal Bisanzio, Nancy Fullman, Chantal K. Huynh, Xie Kulikoff, Michael J. Kutz, Alan D. Lopez, Ali H. Mokdad, Mohsen Naghavi, Grant Nguyen, Katya Anne Shackelford, Theo Vos, Haidong Wang, Stephen S. Lim, Christopher J. L. Murray, Ric N. Price, J. Kevin Baird, David L. Smith, Samir Bhatt, Daniel J. Weiss, Simon I. Hay, and Peter W. Gething. 2019. 'Mapping the global endemicity and clinical burden of Plasmodium vivax, 2000-17: a spatial and temporal modelling study', The Lancet, 394: 332-43.

Boonkaew, Tippawan, Watcharakorn Mongkol, Sureerat Prasert, Pattaweeya Paochan, Saki Yoneda, Wang Nguitragool, Chalermpon Kumpitak, Jetsumon Sattabongkot, and Anchanee Kubera. 2020. 'Transcriptome analysis of Anopheles dirus and Plasmodium vivax at ookinete and oocyst stages', Acta Tropica, 207: 105502.

Boudin, C, A Diop, A Gaye, L Gadiaga, C Gouagna, I Safeukui, and S Bonnet. 2005. 'Plasmodium falciparum transmission blocking immunity in three areas with perennial or seasonal endemicity and different levels of transmission', Am J Trop Med Hyg, 73: 1090 95.

Bourgard, Catarina, Letusa Albrecht, Ana C. A. V. Kayano, Per Sunnerhagen, and Fabio T. M. Costa. 2018. 'Plasmodium vivax Biology: Insights Provided by Genomics, Transcriptomics and Proteomics', Frontiers in Cellular and Infection Microbiology, 8.

Bray, N. L., H. Pimentel, P. Melsted, and L. Pachter. 2016. 'Erratum: Near-optimal probabilistic RNA-seq quantification', Nat Biotechnol, 34: 888. 
Bryant, D. M., K. Johnson, T. DiTommaso, T. Tickle, M. B. Couger, D. Payzin-Dogru, T. J. Lee, N. D. Leigh, T. H. Kuo, F. G. Davis, J. Bateman, S. Bryant, A. R. Guzikowski, S. L. Tsai, S. Coyne, W. W. Ye, R. M. Freeman, Jr., L. Peshkin, C. J. Tabin, A. Regev, B. J. Haas, and J. L. Whited. 2017. 'A Tissue-Mapped Axolotl De Novo Transcriptome Enables Identification of Limb Regeneration Factors', Cell Rep, 18: 762-76.

Camacho, C., G. Coulouris, V. Avagyan, N. Ma, J. Papadopoulos, K. Bealer, and T. L. Madden. 2009. 'BLAST+: architecture and applications', BMC Bioinformatics, 10: 421.

Chen, S., Y. Zhou, Y. Chen, and J. Gu. 2018. 'fastp: an ultra-fast all-in-one FASTQ preprocessor', Bioinformatics, 34: i884-i90.

Dinglasan, R. R., D. E. Kalume, S. M. Kanzok, A. K. Ghosh, O. Muratova, A. Pandey, and M. Jacobs-Lorena. 2007. 'Disruption of Plasmodium falciparum development by antibodies against a conserved mosquito midgut antigen', Proc Natl Acad Sci U S A, 104: 13461-6.

Dong, Y., R. Aguilar, Z. Xi, E. Warr, E. Mongin, and G. Dimopoulos. 2006. 'Anopheles gambiae immune responses to human and rodent Plasmodium parasite species', PLoS Pathog, 2: e52.

Duffy, Patrick E. 2021. 'Transmission-Blocking Vaccines: Harnessing Herd Immunity for Malaria Elimination', Expert Review of Vaccines: null-null.

Ewels, P., M. Magnusson, S. Lundin, and M. Kaller. 2016. 'MultiQC: summarize analysis results for multiple tools and samples in a single report', Bioinformatics, 32: 3047-8.

Fanello, C., F. Santolamazza, and A. della Torre. 2002. 'Simultaneous identification of species and molecular forms of the Anopheles gambiae complex by PCR-RFLP', Med Vet Entomol, 16: 461-4.

Finn, R. D., J. Clements, and S. R. Eddy. 2011. 'HMMER web server: interactive sequence similarity searching', Nucleic Acids Res, 39: W29-37.

Gouagna, L. C., B. Mulder, E. Noubissi, T. Tchuinkam, J. P. Verhave, and C. Boudin. 1998. 'The early sporogonic cycle of Plasmodium falciparum in laboratory-infected Anopheles gambiae: an estimation of parasite efficacy', Trop Med Int Health, 3: 21-8.

Goupeyou-Youmsi, Jessy, Tsiriniaina Rakotondranaivo, Nicolas Puchot, Ingrid Peterson, Romain Girod, Inès Vigan-Womas, Richard Paul, Mamadou Ousmane Ndiath, and Catherine Bourgouin. 2020. 'Differential contribution of Anopheles coustani and Anopheles arabiensis to the transmission of Plasmodium falciparum and Plasmodium vivax in two neighbouring villages of Madagascar', Parasites \& Vectors, 13: 430.

Grabherr, M. G., B. J. Haas, M. Yassour, J. Z. Levin, D. A. Thompson, I. Amit, X. Adiconis, L. Fan, R. Raychowdhury, Q. Zeng, Z. Chen, E. Mauceli, N. Hacohen, A. Gnirke, N. Rhind, F. di Palma, B. W. Birren, C. Nusbaum, K. Lindblad-Toh, N. Friedman, and A. Regev. 2011. 'Full-length transcriptome assembly from RNA-Seq data without a reference genome', Nat Biotechnol, 29: 644-52.

Howes, R. E., K. E. Battle, K. N. Mendis, D. L. Smith, R. E. Cibulskis, J. K. Baird, and S. I. Hay. 2016. 'Global Epidemiology of Plasmodium vivax', Am J Trop Med Hyg, 95: 15-34.

Howes, R. E., S. A. Mioramalala, B. Ramiranirina, T. Franchard, A. J. Rakotorahalahy, D. Bisanzio, P. W. Gething, P. A. Zimmerman, and A. Ratsimbasoa. 2016. 'Contemporary epidemiological overview of malaria in Madagascar: operational utility of reported routine case data for malaria control planning', Malar J, 15: 502.

Isaacs, A. T., N. Jasinskiene, M. Tretiakov, I. Thiery, A. Zettor, C. Bourgouin, and A. A. James. 2012. 'Transgenic Anopheles stephensi coexpressing single-chain antibodies resist Plasmodium falciparum development', Proc Natl Acad Sci U S A, 109: E1922-30. 
Katoh, Y., B. Ritter, T. Gaffry, F. Blondeau, S. Höning, and P. S. McPherson. 2009. 'The clavesin family, neuron-specific lipid- and clathrin-binding Sec14 proteins regulating lysosomal morphology', Journal of Biological Chemistry, 284: 27646-54.

Krogh, A., B. Larsson, G. von Heijne, and E. L. Sonnhammer. 2001. 'Predicting transmembrane protein topology with a hidden Markov model: application to complete genomes', $J$ Mol Biol, 305: 567-80.

Kumari, Seena, Charu Chauhan, Sanjay Tevatiya, Deepak Singla, Tanwee Das De, Punita Sharma, Tina Thomas, Jyoti Rani, Deepali Savargaonkar, Kailash C. Pandey, Veena Pande, and Rajnikant Dixit. 2021. 'Genetic changes of Plasmodium vivax tempers host tissue-specific responses in Anopheles stephensi', Current Research in Immunology, 2: 12-22.

Lagesen, K., P. Hallin, E. A. Rødland, H. H. Staerfeldt, T. Rognes, and D. W. Ussery. 2007. 'RNAmmer: consistent and rapid annotation of ribosomal RNA genes', Nucleic Acids Res, 35: $3100-8$.

Lavazec, C., C. Boudin, R. Lacroix, S. Bonnet, A. Diop, S. Thiberge, B. Boisson, R. Tahar, and C. Bourgouin. 2007. 'Carboxypeptidases B of Anopheles gambiae as targets for a Plasmodium falciparum transmission-blocking vaccine', Infect Immun, 75: 1635-42.

Lavazec, C., and C. Bourgouin. 2008. 'Mosquito-based transmission blocking vaccines for interrupting Plasmodium development', Microbes Infect, 10: 845-9.

Li, A., J. Zhang, and Z. Zhou. 2014. 'PLEK: a tool for predicting long non-coding RNAs and messenger RNAs based on an improved k-mer scheme', BMC Bioinformatics, 15: 311.

Love, M. I., W. Huber, and S. Anders. 2014. 'Moderated estimation of fold change and dispersion for RNA-seq data with DESeq2', Genome Biol, 15: 550.

Mathias, D. K., J. G. Jardim, L. A. Parish, J. S. Armistead, H. V. Trinh, C. Kumpitak, J. Sattabongkot, and R. R. Dinglasan. 2014. 'Differential roles of an Anopheline midgut GPI-anchored protein in mediating Plasmodium falciparum and Plasmodium vivax ookinete invasion', Infect Genet Evol.

Mead, E. A., M. Li, Z. Tu, and J. Zhu. 2012. 'Translational regulation of Anopheles gambiae mRNAs in the midgut during Plasmodium falciparum infection', BMC Genomics, 13: 366.

Menard, Didier, and Arjen Dondorp. 2017. 'Antimalarial Drug Resistance: A Threat to Malaria Elimination', Cold Spring Harbor Perspectives in Medicine, 7.

Mendes, AM, T Schlegelmilch, A Cohuet, P Awono-Ambene, M De Iorio, D Fontenille, I Morlais, GK Christophides, FC Kafatos, and D Vlachou. 2008. 'Conserved mosquito/parasite interactions affect development of Plasmodium falciparum in Africa', PLoS Pathog, 4: e1000069.

Miller, Louis H., Mary H. McGinniss, Paul V. Holland, and Paddy Sigmon. 1978. 'The Duffy Blood Group Phenotype in American Blacks Infected with Plasmodium Vivax in Vietnam', The American Journal of Tropical Medicine and Hygiene, 27: 1069-72.

Mukhtar, Maowia M., Omer A. Eisawi, Seth A. Amanfo, Elwaleed M. Elamin, Zeinab S. Imam, Faiza M. Osman, and Manasik E. Hamed. 2019. 'Plasmodium vivax cerebral malaria in an adult patient in Sudan', Malaria Journal, 18: 316.

Nguyen, Michele, Rosalind E. Howes, Tim C. D. Lucas, Katherine E. Battle, Ewan Cameron, Harry S. Gibson, Jennifer Rozier, Suzanne Keddie, Emma Collins, Rohan Arambepola, Su Yun Kang, Chantal Hendriks, Anita Nandi, Susan F. Rumisha, Samir Bhatt, Sedera A. Mioramalala, Mauricette Andriamananjara Nambinisoa, Fanjasoa Rakotomanana, Peter 
W. Gething, and Daniel J. Weiss. 2020. 'Mapping malaria seasonality in Madagascar using health facility data', BMC Medicine, 18: 26.

Niu, Guodong, Caio França, Genwei Zhang, Wanlapa Roobsoong, Wang Nguitragool, Xiaohong Wang, Jetsumon Prachumsri, Noah S. Butler, and Jun Li. 2017. 'The fibrinogen-like domain of FREP1 protein is a broad-spectrum malaria transmission-blocking vaccine antigen', Journal of Biological Chemistry, 292: 11960-69.

Petersen, T. N., S. Brunak, G. von Heijne, and H. Nielsen. 2011. 'SignalP 4.0: discriminating signal peptides from transmembrane regions', Nat Methods, 8: 785-6.

Rabinovich, R. N., C. Drakeley, A. A. Djimde, B. F. Hall, S. I. Hay, J. Hemingway, D. C. Kaslow, A. Noor, F. Okumu, R. Steketee, M. Tanner, T. N. C. Wells, M. A. Whittaker, E. A. Winzeler, D. F. Wirth, K. Whitfield, and P. L. Alonso. 2017. 'malERA: An updated research agenda for malaria elimination and eradication', PLoS Med, 14: e1002456.

Rahimi, Bilal Ahmad, Ammarin Thakkinstian, Nicholas J. White, Chukiat Sirivichayakul, Arjen M. Dondorp, and Watcharee Chokejindachai. 2014. 'Severe vivax malaria: a systematic review and meta-analysis of clinical studies since 1900', Malaria Journal, 13: 481.

Ramasamy, M. S., R. Kulasekera, K. A. Srikrishnaraj, and R. Ramasamy. 1996. 'Different effects of modulation of mosquito (Diptera:Culicidae) trypsin activity on the infectivity of two human malaria (Hemosporidia:Plasmodidae) parasites', Journal of Medical Entomology, 33: 777-82.

Raquin, Vincent, Sarah Hélène Merkling, Valérie Gausson, Isabelle Moltini-Conclois, Lionel Frangeul, Hugo Varet, Marie-Agnès Dillies, Maria-Carla Saleh, and Louis Lambrechts. 2017. 'Individual co-variation between viral RNA load and gene expression reveals novel host factors during early dengue virus infection of the Aedes aegypti midgut', PLOS Neglected Tropical Diseases, 11: e0006152.

Santana, Rosa Amélia Gonçalves, Maurício Costa Oliveira, Iria Cabral, Rubens Celso Andrade Silva Junior, Débora Raysa Teixeira de Sousa, Lucas Ferreira, Marcus Vinícius Guimarães Lacerda, Wuelton Marcelo Monteiro, Patrícia Abrantes, Maria das Graças Vale Barbosa Guerra, and Henrique Silveira. 2019. 'Anopheles aquasalis transcriptome reveals autophagic responses to Plasmodium vivax midgut invasion', Parasites \& Vectors, 12: 261.

Saul, A. 2007. 'Mosquito stage, transmission blocking vaccines for malaria', Curr Opin Infect Dis, 20: 476-81.

Serrato-Salas, J., J. Izquierdo-Sánchez, M. Argüello, R. Conde, A. Alvarado-Delgado, and H. Lanz-Mendoza. 2018. 'Aedes aegypti antiviral adaptive response against DENV-2', Dev Comp Immunol, 84: 28-36.

Service, M. W. 1993. Mosquito ecology : field sampling methods (Elsevier Applied Science : Elsevier: London; New York ).

Shahabuddin, M., F. Lemos, M. Jacobs-Lorena, and D.C. Kaslow. 1994. "Mechanism of mosquito peritrophic matrix invasion by Plasmodium ookinetes and putative malaria transmission-blocking targets." In 43rd Annual Meeting of the American Society of Tropical Medicine and Hygiene, 128. Cincinnati, Ohio, Nov13-17: Am. J. trop. Med. Hyg.

Sieber, K-P., M. Huber, D. Kaslow, S.M. Banks, M. Torii, M. Aikawa, and L.H. Miller. 1991. 'The peritrophic membrane as a barrier : its penetration by Plasmodium gallinaceum and the effect of a monoclonal antibody to ookinetes', Experimental Parasitology, 72: 145-56. 
Simão, F. A., R. M. Waterhouse, P. Ioannidis, E. V. Kriventseva, and E. M. Zdobnov. 2015. 'BUSCO: assessing genome assembly and annotation completeness with single-copy orthologs', Bioinformatics, 31: 3210-2.

Smith, Ryan C, Joel Vega-Rodríguez, and Marcelo Jacobs-Lorena. 2014. 'The Plasmodium bottleneck: malaria parasite losses in the mosquito vector', Memórias do Instituto Oswaldo Cruz, 109: 644-61.

Solomon, Absra, Daniel Kahase, and Mihret Alemayehu. 2020. 'Trend of malaria prevalence in Wolkite health center: an implication towards the elimination of malaria in Ethiopia by 2030', Malaria Journal, 19: 112.

Soneson, C., M. I. Love, and M. D. Robinson. 2015. 'Differential analyses for RNA-seq: transcript-level estimates improve gene-level inferences', F1000Res, 4: 1521.

Sterkel, Marcos, Hugo D Perdomo, Melina G Guizzo, Ana Beatriz F Barletta, Rodrigo D Nunes, Felipe A Dias, Marcos H F. Sorgine, and Pedro L Oliveira. 2016. 'Tyrosine Detoxification Is an Essential Trait in the Life History of Blood-Feeding Arthropods', Current Biology, 26: 2188-93.

Taylor, E. A., A. Rinaldo-Matthis, L. Li, M. Ghanem, K. Z. Hazleton, M. B. Cassera, S. C. Almo, and V. L. Schramm. 2007. 'Anopheles gambiae purine nucleoside phosphorylase: catalysis, structure, and inhibition', Biochemistry, 46: 12405-15.

Tchuinkam, T., B. Mulder, K. Dechering, H. Stoffels, J.P. Verhave, M. Cot, P. Carnevale, J.H.E.T. Meuwissen, and V. Robert. 1993. 'Experimental Infections of AnophelesGambiae with Plasmodium-Falciparum of Naturally Infected Gametocyte Carriers in Cameroon - Factors Influencing the Infectivity to Mosquitoes', Tropical Medicine and Parasitology, 44: 271-76.

Tecle, Eillen, Crystal B. Chhan, Latisha Franklin, Ryan S. Underwood, Wendy Hanna-Rose, and Emily R. Troemel. 2021. 'The purine nucleoside phosphorylase pnp-1 regulates epithelial cell resistance to infection in C. elegans', PLOS Pathogens, 17: e1009350.

Wampfler, R., F. Mwingira, S. Javati, L. Robinson, I. Betuela, P. Siba, H. P. Beck, I. Mueller, and I. Felger. 2013. 'Strategies for detection of Plasmodium species gametocytes', PLoS ONE, 8: e76316.

Wang, L., H. J. Park, S. Dasari, S. Wang, J. P. Kocher, and W. Li. 2013. 'CPAT: CodingPotential Assessment Tool using an alignment-free logistic regression model', Nucleic Acids Res, 41: e74.

Weiss, Daniel J., Tim C. D. Lucas, Michele Nguyen, Anita K. Nandi, Donal Bisanzio, Katherine E. Battle, Ewan Cameron, Katherine A. Twohig, Daniel A. Pfeffer, Jennifer A. Rozier, Harry S. Gibson, Puja C. Rao, Daniel Casey, Amelia Bertozzi-Villa, Emma L. Collins, Ursula Dalrymple, Naomi Gray, Joseph R. Harris, Rosalind E. Howes, Sun Yun Kang, Suzanne H. Keddie, Daniel May, Susan Rumisha, Michael P. Thorn, Ryan Barber, Nancy Fullman, Chantal K. Huynh, Xie Kulikoff, Michael J. Kutz, Alan D. Lopez, Ali H. Mokdad, Mohsen Naghavi, Grant Nguyen, Katya Anne Shackelford, Theo Vos, Haidong Wang, David L. Smith, Stephen S. Lim, Christopher J. L. Murray, Samir Bhatt, Simon I. Hay, and Peter W. Gething. 2019. 'Mapping the global prevalence, incidence, and mortality of Plasmodium falciparum, 2000-17: a spatial and temporal modelling study', The Lancet, 394: 322-31.

WHO. 2020. 'World Malaria Report 2020'.

Young, M. D., M. J. Wakefield, G. K. Smyth, and A. Oshlack. 2010. 'Gene ontology analysis for RNA-seq: accounting for selection bias', Genome Biol, 11: R14. 
bioRxiv preprint doi: https://doi.org/10.1101/2021.05.28.446219; this version posted May 28, 2021. The copyright holder for this preprint (which

was not certified by peer review) is the author/funder, who has granted bioRxiv a license to display the preprint in perpetuity. It is made available under aCC-BY-NC-ND 4.0 International license.

Zhang, G., G. Niu, C. M. Franca, Y. Dong, X. Wang, N. S. Butler, G. Dimopoulos, and J. Li. 2015. 'Anopheles Midgut FREP1 Mediates Plasmodium Invasion', Journal of Biological Chemistry, 290: 16490-501. 
Table 1

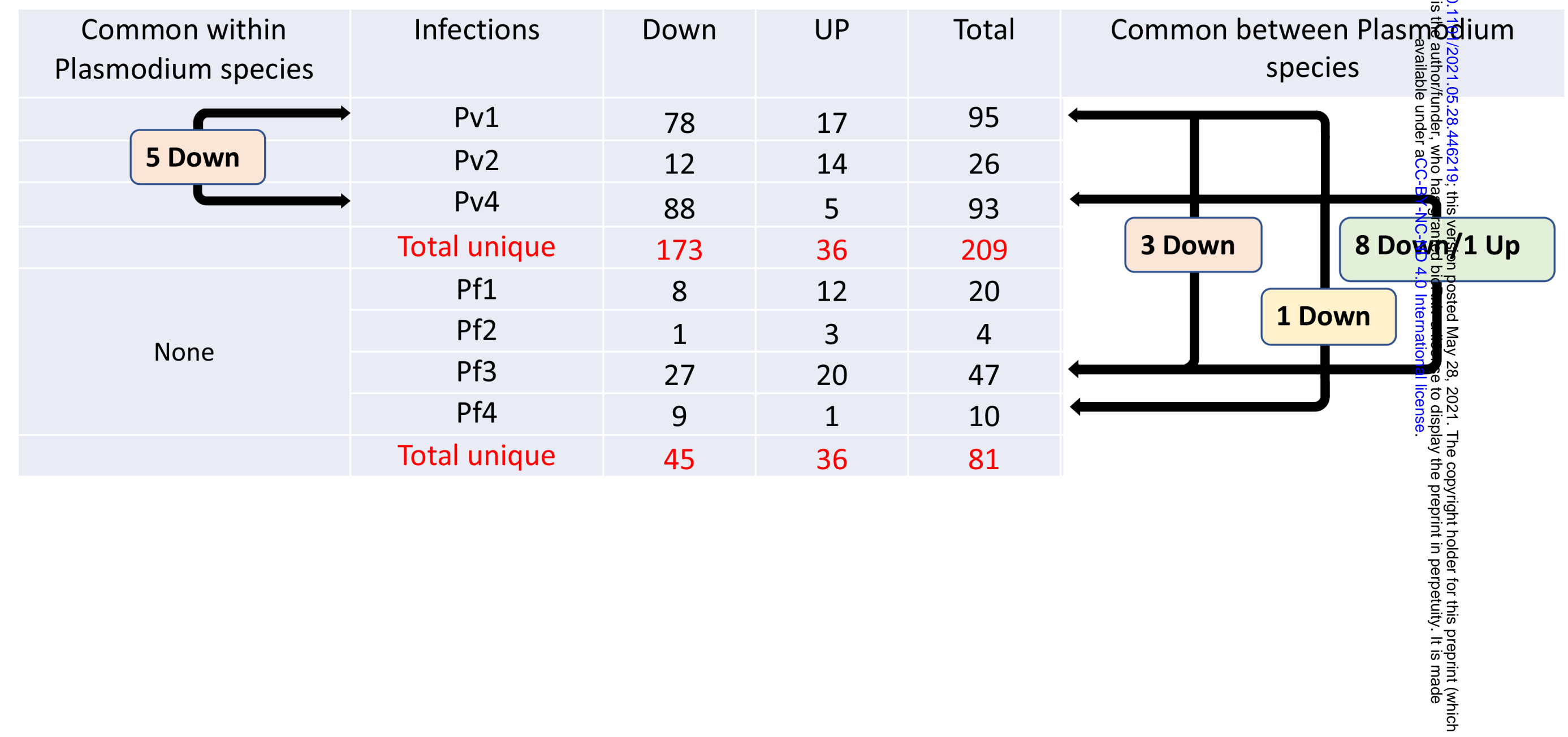


$\mathbf{A}$

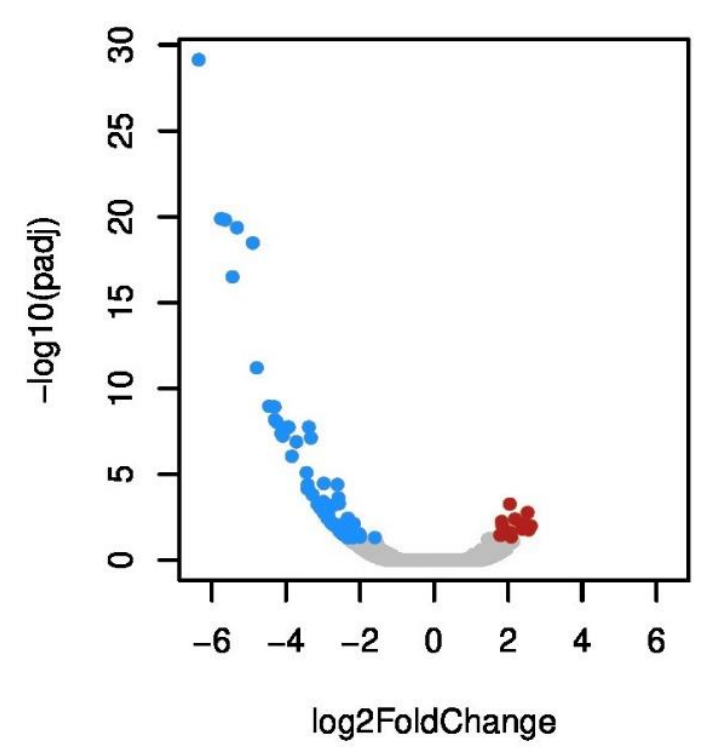

B

FIGURE 1
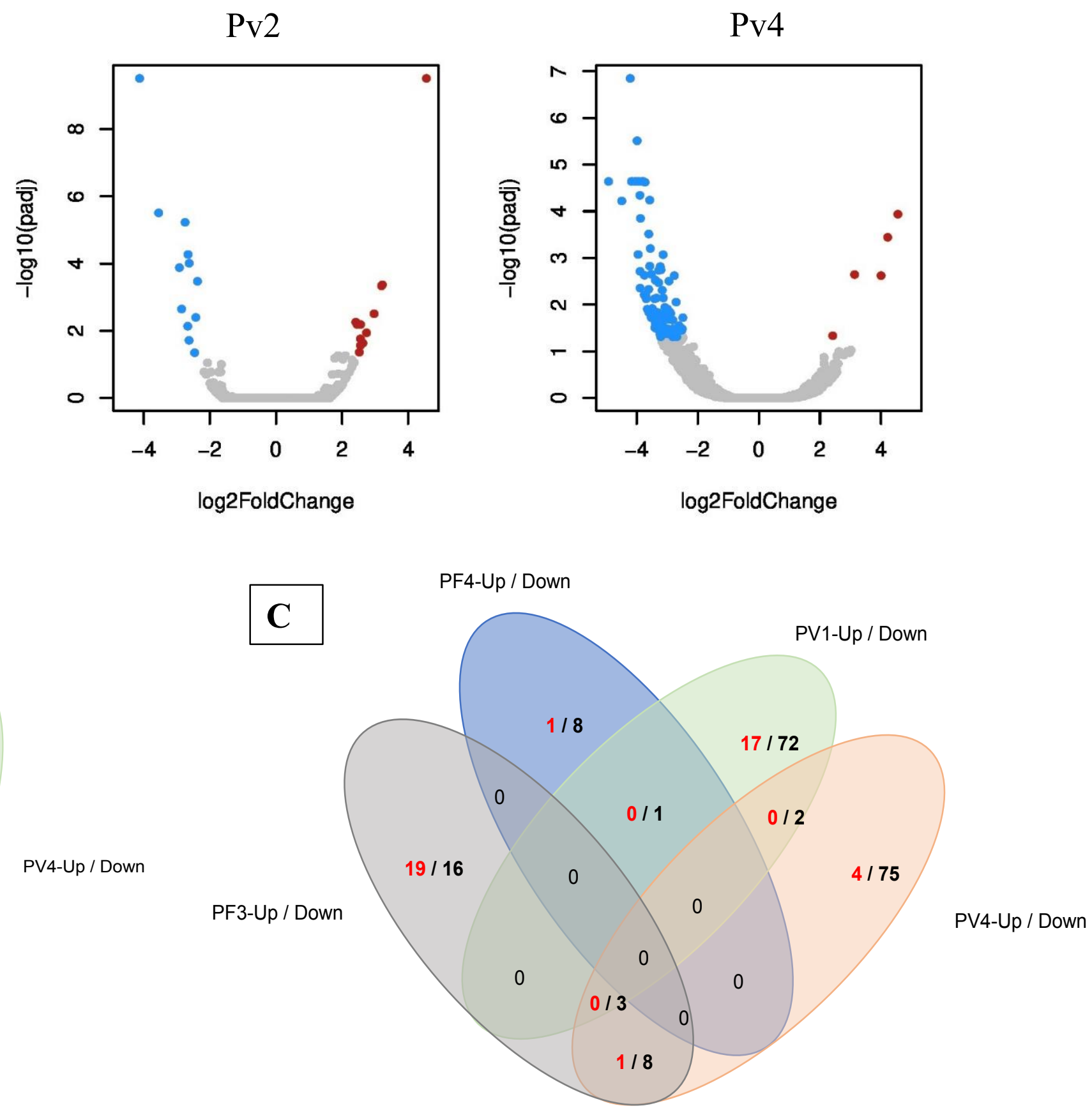

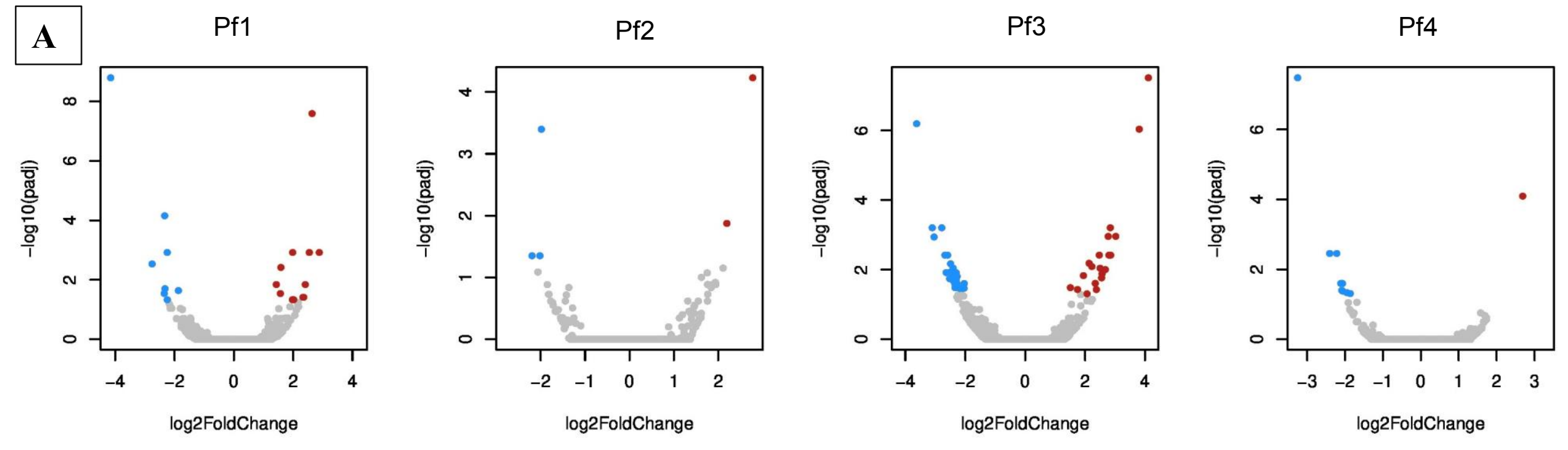

B

PF2-Up / Down

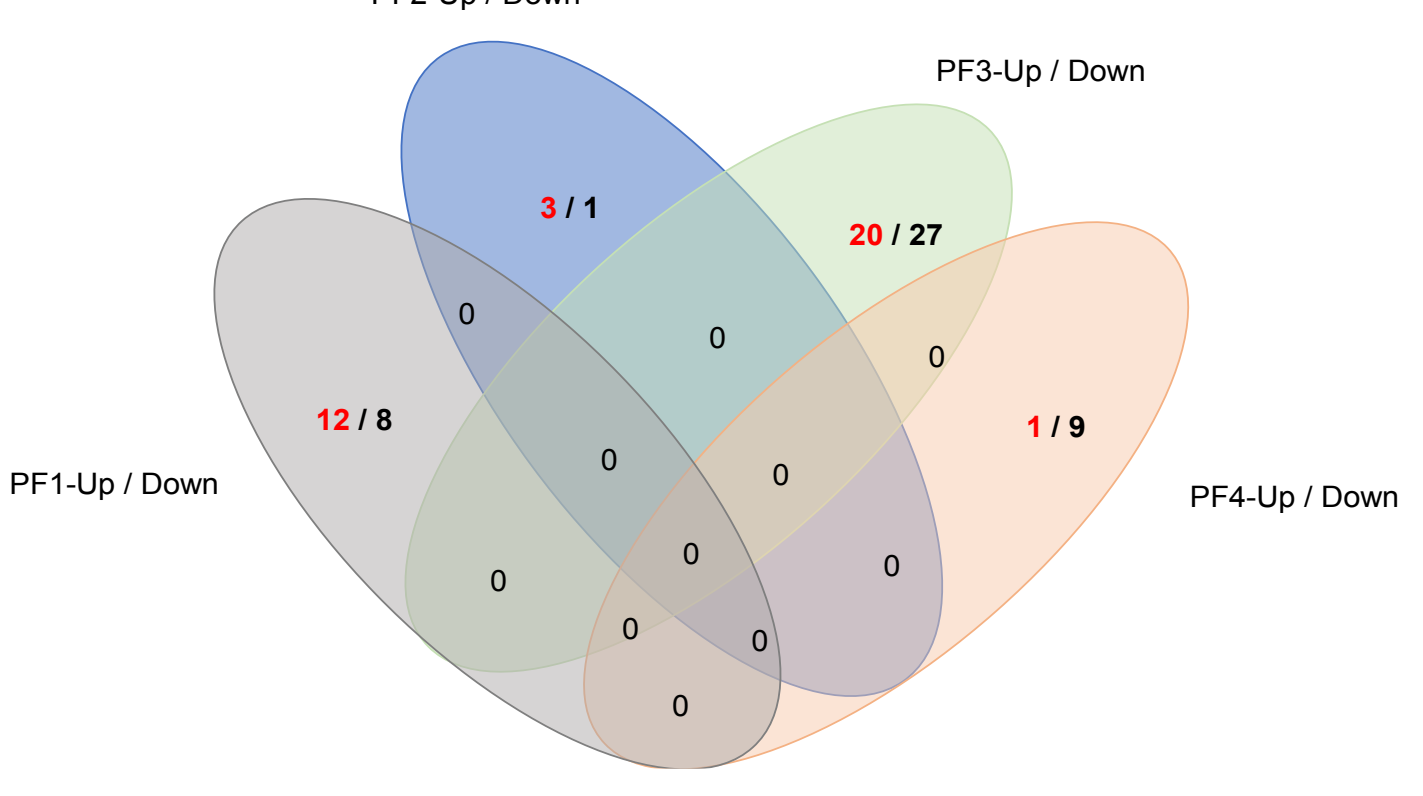

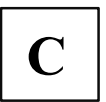

PF1-Up/Down

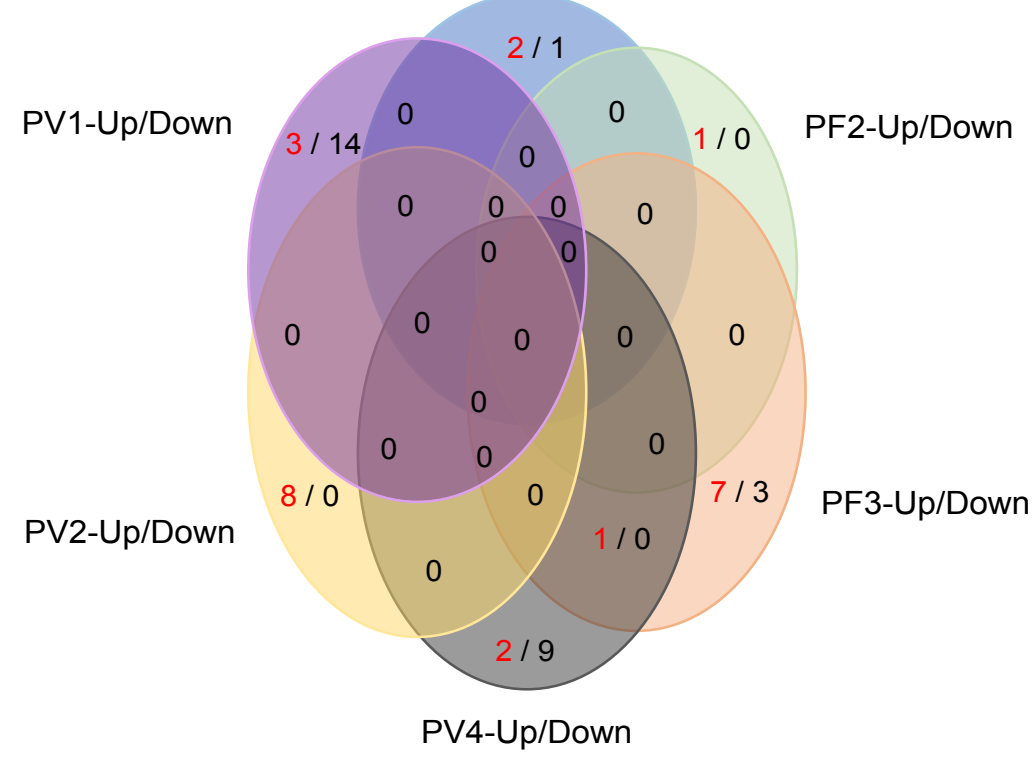

\section{FIGURE 2}




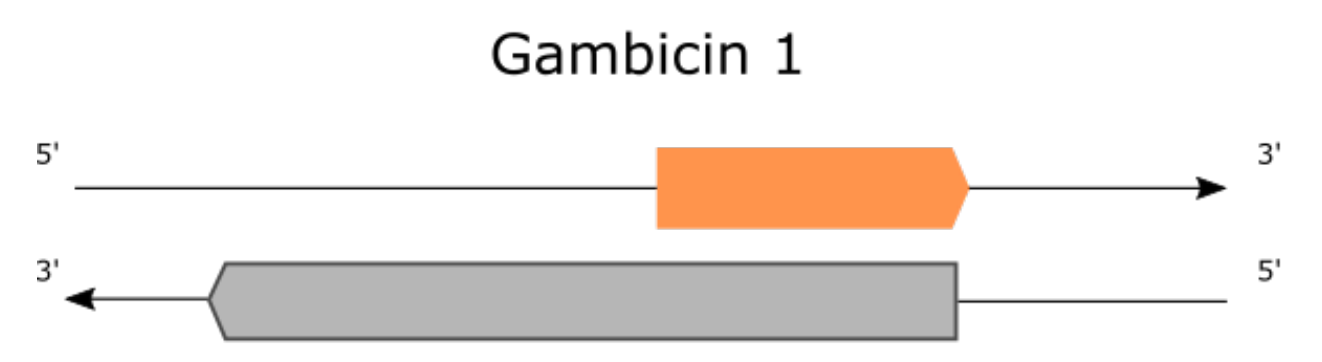

Peptidoglycan recognition protein

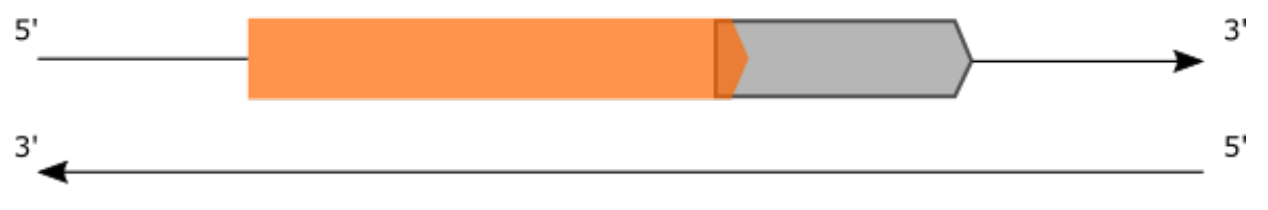

Leucine rich repeat protein

Leucine rich immune protein
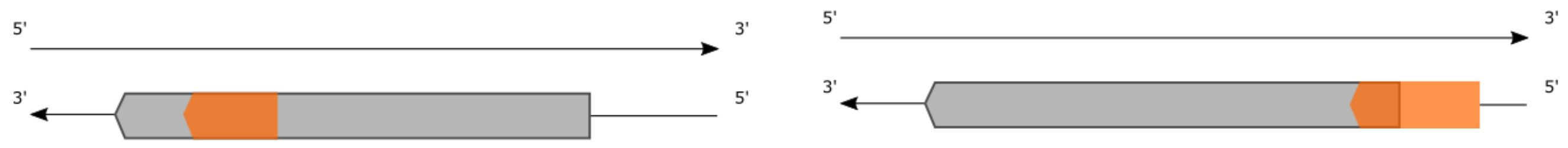

Coding gene

IncRNA

\section{FIGURE 3}




\section{A}

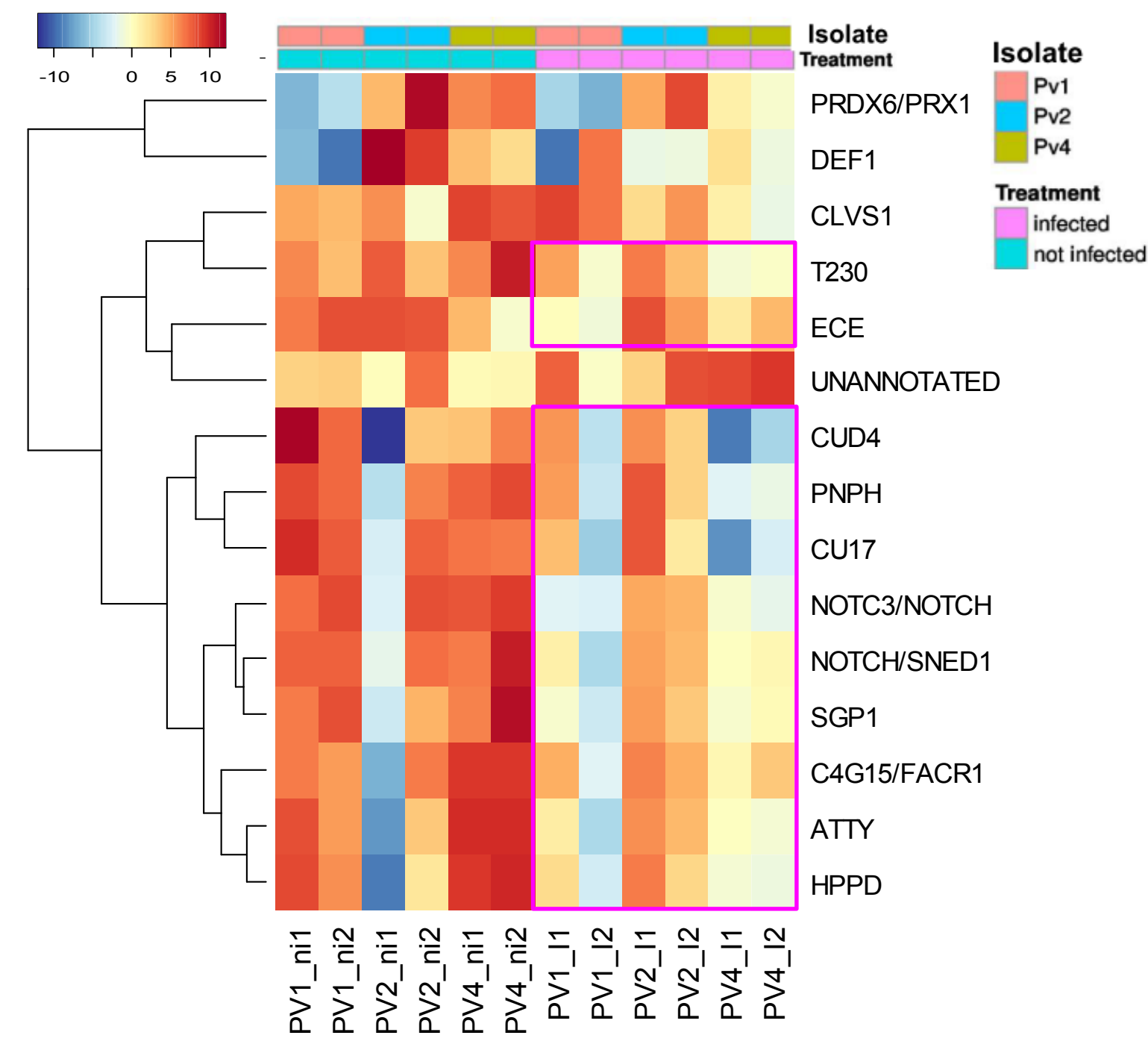

B

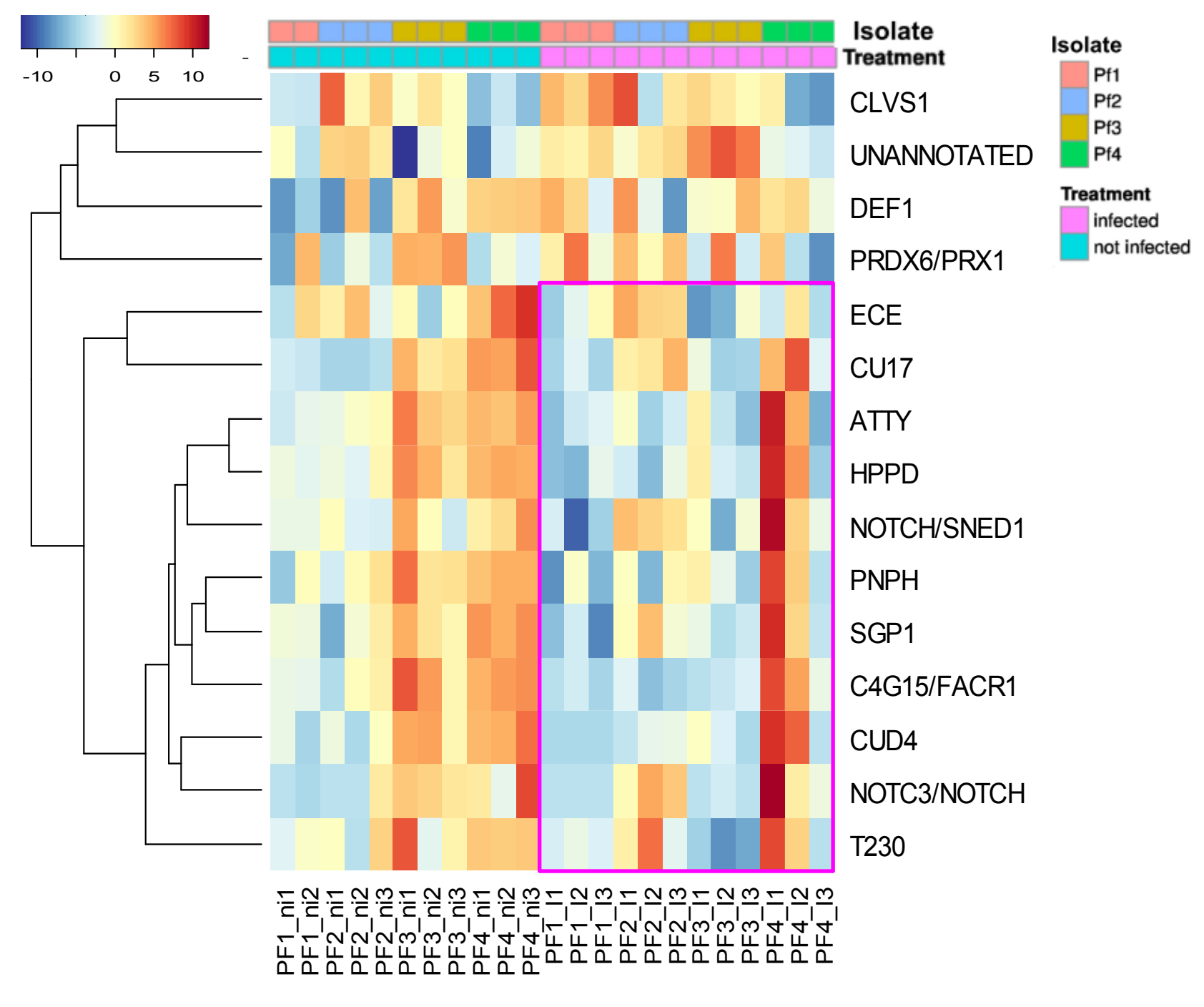

\section{FIGURE 4}


A

organic acid metabolic process
organonitrogen compound biosythes ound metabolic process

heterocycle biosynthetetic proces

cellular nitrogen compound metabolic process

organic substance biosynthetic proces

cellular biosynthetic proces

small molecule metabolic process

primary metabolic proces

organic cyclic compound metabolic proces
organonitrogen compound metabolic proces

organic substance metabolic process

nitrogen compound metabolic proces

cellular process
metabolic process

metabolic process
biological_process

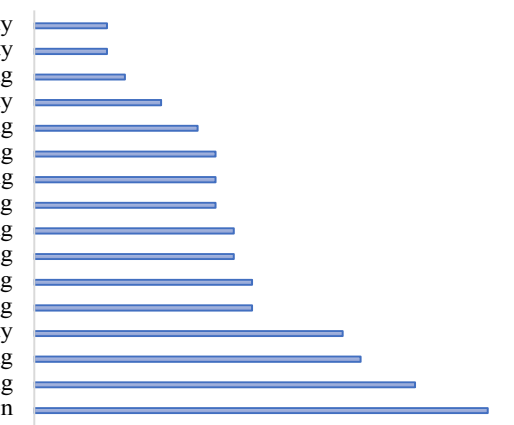

ए
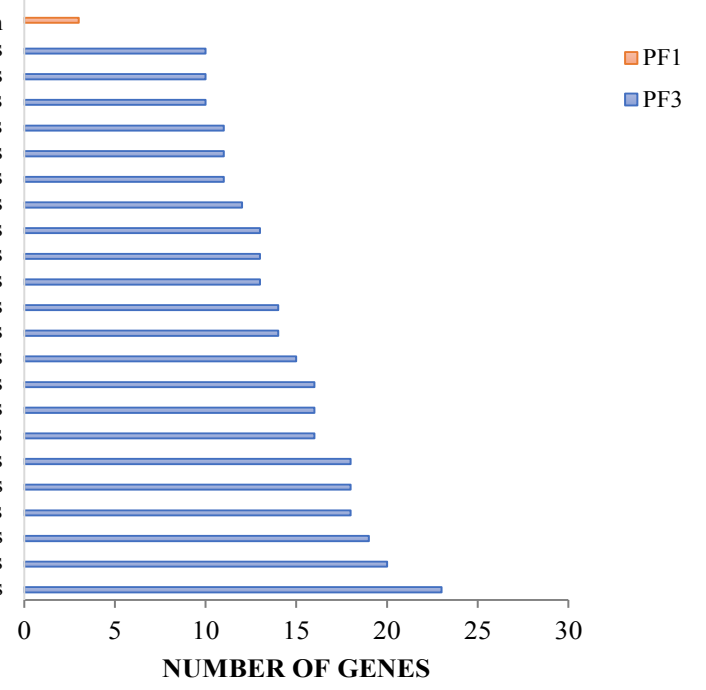

B

BIOLOGICAL PROCESS
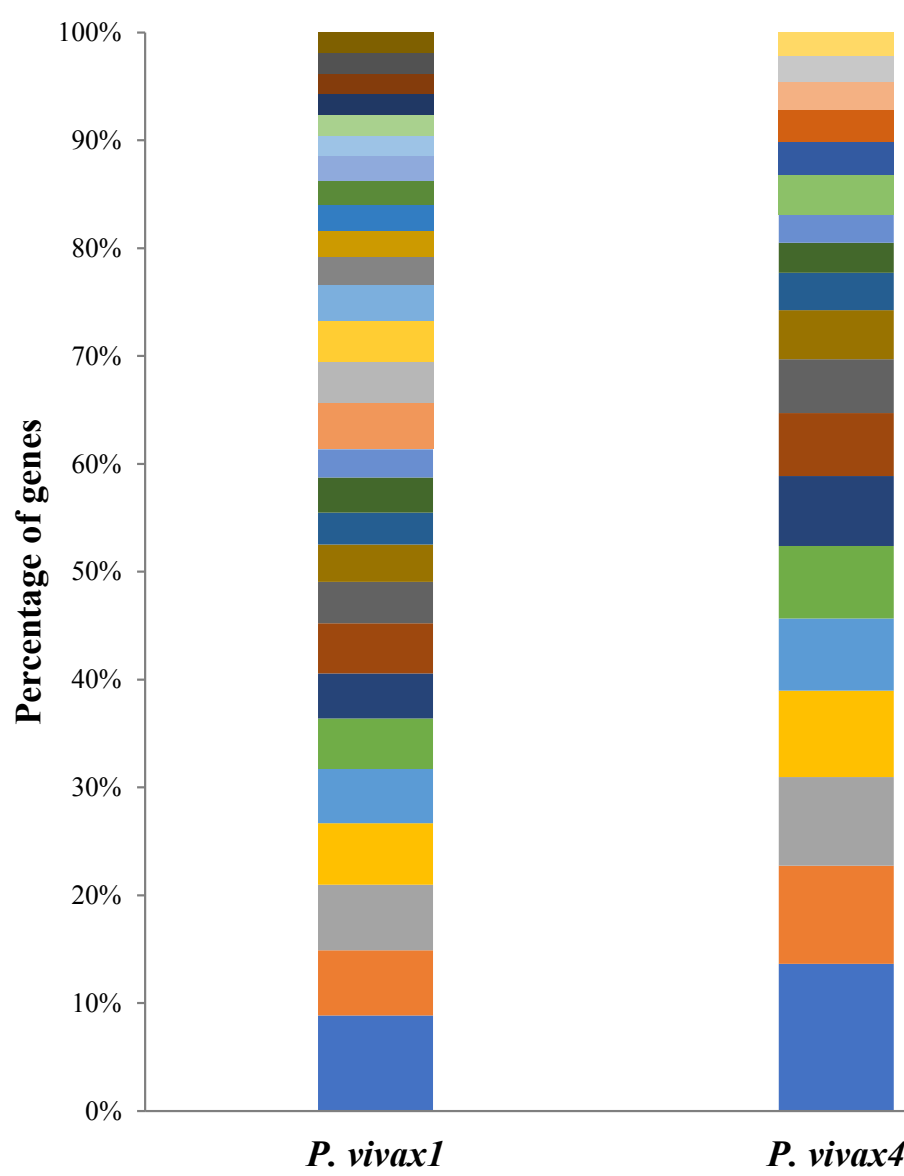

- regulation of biological quality

- cellular developmental process

- regulation of multicellular organismal process

- regulation of developmental process

$n$ response to external stimulus

multi-organism process

- organic substance catabolic process

organic acid metabolic process

$\square$ cellular catabolic process

- localization

- reproductive process

- cellular component organization or biogenesis

- cellular component organization

- anatomical structure development

- small molecule metabolic process

- catabolic process

- cellular aromatic compound metabolic process

$\square$ regulation of cellular process

- developmental process

- multicellular organismal process

macromolecule metabolic process

- proteolysis

\section{FIGURE 5 A-B}



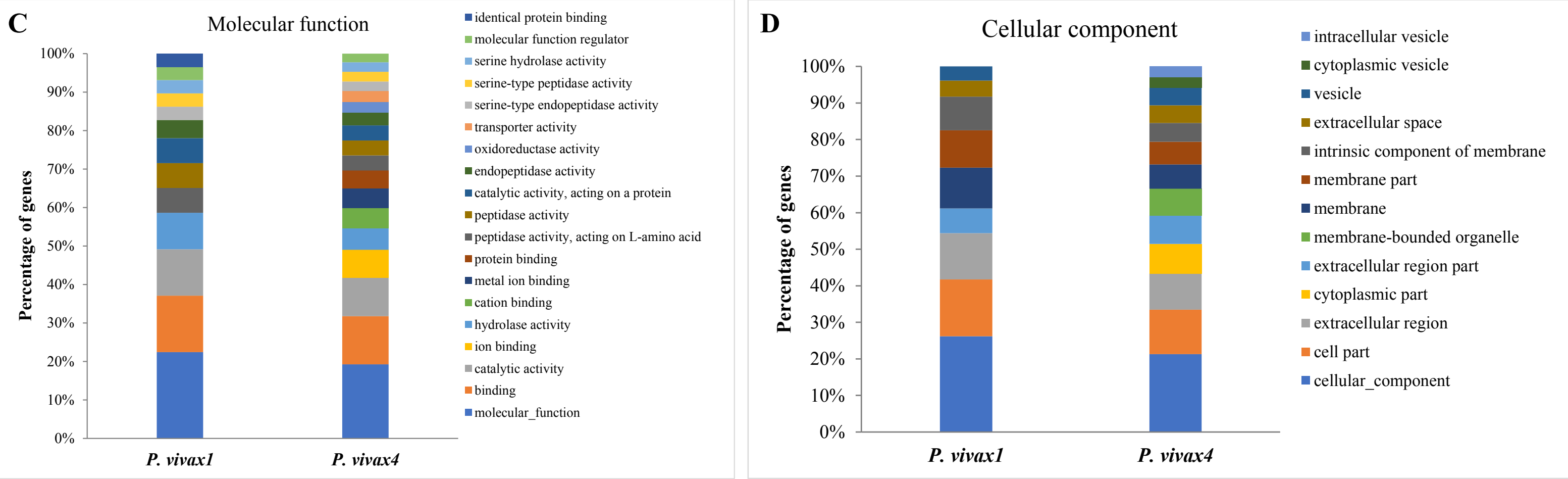

\section{FIGURE 5 C-D}

